\title{
THE BOUNDEDNESS OF MULTILINEAR CALDERÓN-ZYGMUND OPERATORS ON WEIGHTED AND VARIABLE HARDY SPACES
}

\author{
David Cruz-Uribe, Ofs, Kabe Moen, and Hanh Van Nguyen
}

\begin{abstract}
We establish the boundedness of the multilinear Calderón-Zygmund operators from a product of weighted Hardy spaces into a weighted Hardy or Lebesgue space. Our results generalize to the weighted setting results obtained by Grafakos and Kalton [18] and recent work by the third author, Grafakos, Nakamura, and Sawano [20]. As part of our proof we provide a finite atomic decomposition theorem for weighted Hardy spaces, which is interesting in its own right. As a consequence of our weighted results, we prove the corresponding estimates on variable Hardy spaces. Our main tool is a multilinear extrapolation theorem that generalizes a result of the first author and Naibo [10].
\end{abstract}

2010 Mathematics Subject Classification: 42B25, 42B30, $42 B 35$.

Key words: Muckenhoupt weights, weighted Hardy spaces, variable Hardy spaces, multilinear Calderón-Zygmund operators, singular integrals.

\section{Introduction}

In this paper we study the boundedness of multilinear Calderón-Zygmund operators ( $m$-CZOs) on products of weighted and variable Hardy spaces. More precisely, we are interested in the following operators. Let $K\left(y_{0}, y_{1}, \ldots, y_{m}\right)$ be a kernel that is defined away from the diagonal $y_{0}=y_{1}=\cdots=y_{m}$ in $\left(\mathbb{R}^{n}\right)^{m+1}$ and satisfies the smoothness condition

$$
\begin{aligned}
& \left|\partial_{y_{0}}^{\alpha_{0}} \cdots \partial_{y_{m}}^{\alpha_{m}} K\left(y_{0}, y_{1}, \ldots, y_{m}\right)\right| \\
& \quad \leq A_{\alpha_{0}, \ldots, \alpha_{m}}\left(\sum_{k, l=0}^{m}\left|y_{k}-y_{l}\right|\right)^{-\left(m n+\left|\alpha_{0}\right|+\cdots+\left|\alpha_{m}\right|\right)}
\end{aligned}
$$

The first author is supported by NSF Grant DMS-1362425 and research funds from the Dean of the College of Arts \& Sciences, the University of Alabama. The second author is supported by the Simons Foundation. 
for all $\alpha=\left(\alpha_{0}, \ldots, \alpha_{m}\right)$ such that $|\alpha|=\left|\alpha_{0}\right|+\cdots+\left|\alpha_{m}\right| \leq N$, where $N$ is a sufficiently large integer. An $m$-CZO is a multilinear operator $T$ that satisfies

$$
T: L^{q_{1}}\left(\mathbb{R}^{n}\right) \times \cdots \times L^{q_{m}}\left(\mathbb{R}^{n}\right) \rightarrow L^{q}\left(\mathbb{R}^{n}\right)
$$

for some $1<q_{1}, \ldots, q_{m}<\infty$ and $\frac{1}{q}=\frac{1}{q_{1}}+\cdots+\frac{1}{q_{m}}$, and $T$ has the integral representation

$$
T\left(f_{1}, \ldots, f_{m}\right)(x)=\int_{\left(\mathbb{R}^{n}\right)^{m}} K\left(x, y_{1}, \ldots, y_{m}\right) f\left(y_{1}\right) \cdots f\left(y_{m}\right) d y_{1} \cdots d y_{m}
$$

whenever $f_{i} \in L_{c}^{\infty}\left(\mathbb{R}^{n}\right)$ and $x \notin \cap_{i} \operatorname{supp}\left(f_{i}\right)$.

Multilinear CZOs were introduced by Coifman and Meyer $[\mathbf{2}, \mathbf{3}]$ in the 1970s and were systematically studied by Grafakos and Torres [22]. They showed that $m$-CZOs are bounded from $L^{p_{1}}\left(\mathbb{R}^{n}\right) \times \cdots \times L^{p_{m}}\left(\mathbb{R}^{n}\right) \rightarrow$ $L^{p}\left(\mathbb{R}^{n}\right)$, for any $1<p_{1}, \ldots, p_{m}<\infty$ and $p$ defined by $\frac{1}{p}=\frac{1}{p_{1}}+\cdots+\frac{1}{p_{m}}$. Further, $m$-CZOs satisfy weak endpoint bounds when $p_{i}=1$ for some $i$. For Lebesgue space bounds, it is sufficient to take $N=1$ in (1.1) and in fact weaker regularity conditions are sufficient. Bounds for $m$-CZOs from products of Hardy spaces into Lebesgue spaces were proved by Grafakos and Kalton [18] (see also Grafakos and He [17]). As in the linear case, more regularity is required on the operators: in this case, $N \geq s=\left\lfloor n\left(\frac{1}{p}-1\right)\right\rfloor_{+}$where $x_{+}=\max (0, x)$. Very recently, bounds into Hardy spaces were proved by the third author, Grafakos, Nakamura, and Sawano $[\mathbf{2 0}]$. To map into Hardy spaces the kernel $K$ must satisfy (1.1) for

$$
N>s+\max \left\{\left\lfloor m n\left(\frac{1}{p_{k}}-1\right)\right\rfloor_{+}: 1 \leq k \leq m\right\}+m n .
$$

Moreover, in the multilinear case the operator $T$ must satisfy an additional cancelation condition:

$$
\int x^{\alpha} T\left(a_{1}, \ldots, a_{m}\right)(x) d x=0,
$$

for all $|\alpha| \leq s$ and all $\left(p_{k}, \infty, N\right)$ atoms $a_{k}$. For linear CZOs of convolution type, this condition holds automatically: see [21, Lemma 2.1]. An example of a bilinear CZO that satisfies this cancelation condition is $T=R_{1}+R_{2}$, where $R_{i}$ is the bilinear Riesz transform

$$
R_{i}(f, g)(x)=\text { p.v. } \int_{\mathbb{R}} \int_{\mathbb{R}} \frac{x-y_{i}}{\left|\left(x-y_{1}, x-y_{2}\right)\right|^{3}} f\left(y_{1}\right) g\left(y_{2}\right) d y_{1} d y_{2} .
$$


Somewhat surprisingly, neither Riesz transform itself has sufficient cancellation. For more examples of convolution-type multilinear operators that do and do not satisfy this cancelation condition, see $\left[\mathbf{2 0 , 2 1}\right.$. $^{1}$

Weighted norm inequalities for multilinear operators were first considered by Grafakos and Torres [23]. Later, Lerner et al. [25] characterized the weighted inequalities for $m$-CZOs using a multilinear generalization of the Muckenhoupt $A_{p}$ condition. Weighted Hardy spaces were introduced by García-Cuerva [14]. A complete treatment of weighted Hardy spaces is due to Strömberg and Torchinsky [31]; they proved that (linear) Calderón-Zygmund operators whose kernels have enough regularity map $H^{p}(w)$ into $L^{p}(w)$ or $H^{p}(w)$, for $0<p<\infty$ and for weights $w \in A_{\infty}$.

Our goal is to generalize the results of Strömberg and Torchinsky to $m$-CZOs. To state them, we first define some notation. To do so we rely on some (hopefully) well-known concepts; complete definitions will be given below. Given $w \in A_{\infty}$, we define

$$
r_{w}=\inf \left\{r \in(1, \infty): w \in A_{r}\right\}
$$

and for $0<p<\infty$ we define the critical index $s_{w}$ of $w$ by

$$
s_{w}=\left\lfloor n\left(\frac{r_{w}}{p}-1\right)\right\rfloor_{+} .
$$

Our first result gives the boundedness of $m$-CZOs into weighted Lebesgue spaces.

Theorem 1.1. Given an integer $m \geq 1,0<p_{1}, \ldots, p_{m}<\infty$, and $w_{k} \in A_{\infty}, 1 \leq k \leq m$, let $T$ be an $m-C Z O$ associated to a kernel $K$ that satisfies (1.1) for $N$ such that

$$
N \geq \max \left\{\left\lfloor m n\left(\frac{r_{w_{k}}}{p_{k}}-1\right)\right\rfloor_{+}, 1 \leq k \leq m\right\}+(m-1) n .
$$

Then

$$
T: H^{p_{1}}\left(w_{1}\right) \times \cdots \times H^{p_{m}}\left(w_{m}\right) \rightarrow L^{p}(\bar{w}),
$$

where $\bar{w}=\prod_{k=1}^{m} w_{k}^{\frac{p}{p_{k}}}$ and

$$
\frac{1}{p}=\frac{1}{p_{1}}+\cdots+\frac{1}{p_{m}} .
$$

Our second result gives boundedness of $m$-CZOs into weighted Hardy spaces.

\footnotetext{
${ }^{1}$ We note in passing that the results for $m$-CZOs in $[\mathbf{2 0}]$ are stated for convolution type operators, but as the authors note (see Remark 3.4), their results extend to non-convolution type $m$-CZOs.
} 
Theorem 1.2. Given $p, p_{1}, \ldots, p_{m}, \bar{w}, w_{1}, \ldots, w_{m}$ and $T$ as in Theorem 1.1, suppose the kernel $K$ satisfies (1.1) for $N$ such that

$$
N>s_{\bar{w}}+\max \left\{\left\lfloor m n\left(\frac{r_{w_{k}}}{p_{k}}-1\right)\right\rfloor_{+}, 1 \leq k \leq m\right\}+m n .
$$

Suppose further that $T$ satisfies the cancellation condition (1.2) for all $|\alpha| \leq s \bar{w}$, where for $1 \leq k \leq m, a_{k}$ is an $(N, \infty)$ atom: i.e., $a_{k}$ is supported on a cube $Q_{k},\left\|a_{k}\right\|_{\infty} \leq 1$, and

$$
\int_{\mathbb{R}^{n}} x^{\beta} a_{k}(x) d x=0
$$

for all $|\beta| \leq N$. Then

$$
T: H^{p_{1}}\left(w_{1}\right) \times \cdots \times H^{p_{m}}\left(w_{m}\right) \rightarrow H^{p}(\bar{w}) .
$$

Remark 1.3. In Theorems 1.1 and 1.2, if all the weights $w_{k}=1$, then $r_{w_{k}}=1$, so we recapture the unweighted results in $[\mathbf{1 8}, \mathbf{2 0}]$.

Remark 1.4. If $p>1$ and $w \in A_{p}$, then $H^{p}(w)=L^{p}(w)$ (see $[\mathbf{3 1}]$ ). Therefore, in Theorems 1.1 and 1.2, if $w_{k} \in A_{p_{k}}$, then we can replace $H^{p_{k}}\left(w_{k}\right)$ by $L^{p_{k}}\left(w_{k}\right)$ in the conclusion.

Remark 1.5. Implicit in the statement of Theorem 1.2 is the assumption that $\bar{w} \in A_{\infty}$. However, this is always the case: see Lemma 2.1 below.

Remark 1.6. Earlier, Xue and Yan [33] proved a version of Theorem 1.1 with the additional restriction that $0<p_{k} \leq 1$ for all $1 \leq k \leq m$. We want to thank the authors for calling our attention to their paper, which we had overlooked.

Our next pair of results are the analogs of Theorems 1.1 and 1.2 for the variable Lebesgue spaces. The variable Lebesgue spaces are a generalization of the classical $L^{p}$ spaces with the exponent $p$ replaced by a measurable exponent function $p(\cdot): \mathbb{R}^{n} \rightarrow(0, \infty)$. It consists of all measurable functions $f$ such that for some $\lambda>0$

$$
\rho(f / \lambda)=\int_{\mathbb{R}^{n}}\left(\frac{|f(x)|}{\lambda}\right)^{p(x)} d x<\infty .
$$

This becomes a quasi-Banach space with quasi-norm

$$
\|f\|_{p}(\cdot)=\inf \{\lambda>0: \rho(f / \lambda) \leq 1\} .
$$

If $p(x) \geq 1$ a.e., then this is a norm and $L^{p(\cdot)}$ is a Banach space. These spaces were introduced by Orlicz [29] in 1931, and have been extensively studied by a number of authors in the past 25 years. For complete details and references, see [6]. Variable Hardy spaces were introduced by the first author and Wang [12] and independently by Nakai and Sawano [28]. 
In variable Lebesgue exponent spaces, harmonic analysis requires some assumption of regularity on the exponent function $p(\cdot)$. A common assumption that is sufficient for almost all applications is that the exponent function is log-Hölder continuous both locally and at infinity. More precisely, there exist constants $C_{0}, C_{\infty}$, and $p_{\infty}$ such that

$$
|p(x)-p(y)| \leq \frac{C_{0}}{-\log (|x-y|)}, \quad 0<|x-y|<\frac{1}{2},
$$

and

$$
\left|p(x)-p_{\infty}\right| \leq \frac{C_{\infty}}{\log (e+|x|)}
$$

Finally, given an exponent function $p(\cdot)$, we define

$$
p_{-}=\underset{x \in \mathbb{R}^{n}}{\operatorname{essinf}} p(x), \quad p_{+}=\underset{x \in \mathbb{R}^{n}}{\operatorname{esssup}} p(x) .
$$

As an immediate application of Theorems 1.1 and 1.2, and multilinear Rubio de Francia extrapolation in the scale of variable Lebesgue spaces, we get the following two results.

Theorem 1.7. Given an integer $m \geq 1$, let $p_{1}, \ldots, p_{m}$ be real numbers, and let $q_{1}(\cdot), \ldots, q_{m}(\cdot)$ be log-Hölder continuous exponent functions such that $0<p_{k}<\left(q_{k}\right)_{-} \leq\left(q_{k}\right)_{+}<\infty$. Define

$$
\frac{1}{q(\cdot)}=\frac{1}{q_{1}(\cdot)}+\cdots+\frac{1}{q_{m}(\cdot)} .
$$

Let $T$ be an $m$-CZO as in Theorem 1.1 satisfying (1.1) for all $|\alpha| \leq N$ with

$$
N \geq \max \left\{\left\lfloor m n\left(\frac{1}{p_{k}}-1\right)\right\rfloor_{+}, 1 \leq k \leq m\right\}+(m-1) n .
$$

Then

$$
T: H^{q_{1}(\cdot)} \times \cdots \times H^{q_{m}(\cdot)} \rightarrow L^{q(\cdot)} .
$$

Theorem 1.8. Given $q(\cdot), q_{1}(\cdot), \ldots, q_{m}(\cdot), p_{1}, \ldots, p_{m}$ as in Theorem 1.7, define $p$ by

$$
\frac{1}{p}=\frac{1}{p_{1}}+\cdots+\frac{1}{p_{m}}
$$

Let $T$ be an $m-C Z O$ as in Theorem 1.1 satisfying (1.1) for all $|\alpha| \leq N$ with

$$
N>\left\lfloor n\left(\frac{1}{p}-1\right)\right\rfloor_{+}+\max \left\{\left\lfloor m n\left(\frac{1}{p_{k}}-1\right)\right\rfloor_{+}, 1 \leq k \leq m\right\}+m n .
$$

Suppose further that $T$ satisfies (1.2) for all $|\alpha| \leq\lfloor n(1 / p-1)\rfloor_{+}$. Then

$$
T: H^{q_{1}(\cdot)} \times \cdots \times H^{q_{m}(\cdot)} \rightarrow H^{q(\cdot)} .
$$


Remark 1.9. We chose to prove Theorems 1.7 and 1.8 using Rubio de Francia extrapolation because of the simplicity of this approach. However, it should be possible to prove these results directly, adapting the unweighted proof in [20] to the variable exponent setting. Many of the technical, weighted results for the proof contained in Sections 2 and 3 also hold in the variable exponent case: see $[\mathbf{1 2}, \mathbf{2 8}, \mathbf{3 0}]$.

As we were completing this paper we learned that this approach was taken by Tan $[\mathbf{3 2}]$. He proved bilinear versions of Theorems 1.7 and 1.8, with the additional hypothesis that $\left(q_{k}\right)_{+} \leq 1$ for all $1 \leq k \leq m$. We want to thank him for sharing with us a preprint of his work.

Remark 1.10. Our techniques should apply to a wider class of operators, namely, we believe that Theorems $1.1,1.2,1.7$, and 1.8 will hold for the classes of product type and mixed type operators defined on pages 2 and 3 of [20].

The remainder of this paper is organized as follows. In Section 2 we give some basic definitions and theorems about weights that we will use in subsequent sections. In particular, we prove a finite atomic decomposition for weighted Hardy spaces that extends the results in [12]. In Section 3 we gather together a number of technical lemmas that we need for the proofs of Theorems 1.1 and 1.2. Then in Sections 4 and 5 we prove these results. Finally, in Section 6 we give some basic facts about variable exponent spaces and prove Theorems 1.7 and 1.8. In fact, we prove more general results which include these theorems as special cases. Their statements, however, require additional facts about variable exponent spaces, and so we delay their statement until the final section.

Throughout this paper, we will use $n$ to denote the dimension of the underlying space, $\mathbb{R}^{n}$, and will use $m$ to denote the "dimension" of our multilinear operators. By a cube $Q$ we will always mean a cube whose sides are parallel to the coordinate axes, and for $\tau>1$ let $\tau Q$ denote the cube with same center such that $\ell(\tau Q)=\tau \ell(Q)$. We define the average of a function $f$ on a cube $Q$ by $f_{Q}=f_{Q} f d x=|Q|^{-1} \int_{Q} f d x$. By $C, c$, etc. we will mean constants that may depend on the underlying parameters in the problem. Sometimes, to emphasize that they (only) depend on certain parameters, we will write $C(X, Y, Z, \ldots)$. The values of these constants may change from line to line. If we write $A \lesssim B$, we mean that $A \leq c B$ for some constant $c$.

\section{Weights and weighted Hardy spaces}

Weights and weighted norm inequalities. In this section we give some basic definitions and results about $A_{p}$ weights. For complete information, we refer the reader to $[\mathbf{1 3}, \mathbf{1 5}, \mathbf{1 6}]$. By a weight $w$ we always 
mean a non-negative, locally integrable function such that $0<w(x)<\infty$ a.e. For $1<p<\infty$, we say that $w$ is in the Muckenhoupt class $A_{p}$, denoted by $w \in A_{p}$, if

$$
[w]_{A_{p}}=\sup _{Q}\left(f_{Q} w d x\right)\left(f_{Q} w^{1-p^{\prime}} d x\right)^{p-1}<\infty .
$$

When $p=1$ we say that $w \in A_{1}$ if there is a constant $C$ such that, for every cube $Q$ and a.e. $x \in Q$,

$$
f_{Q} w d x \leq C w(x)
$$

The infimum over all such constants will be denoted by $[w]_{A_{1}}$. The $A_{p}$ classes are nested: for $1<p<q<\infty, A_{1} \subsetneq A_{p} \subsetneq A_{q}$. Let $A_{\infty}$ denote the union of all the $A_{p}$ classes, $p \geq 1$.

Given $w \in A_{\infty}$, then $w$ is a doubling measure. More precisely, if $w \in A_{p}$ for some $p \geq 1$, then it follows from the definition that given any cube $Q$ and $\tau>1$

$$
w(\tau Q) \leq C \tau^{n p} w(Q)
$$

In the study of multilinear weighted norm inequalities, we often need the fact that the convex hull of $A_{\infty}$ weights is again in $A_{\infty}$. The following result can be found, for instance, in [33] or in [19, Lemma 5]. For completeness we sketch a short proof, using a multilinear reverse Hölder inequality: if $w_{1}, \ldots, w_{m} \in A_{\infty}, 1<p_{1}, \ldots, p_{m}<\infty$, and $\frac{1}{p}=\frac{1}{p_{1}}+\cdots+$ $\frac{1}{p_{m}}$, then for every cube $Q$

$$
\prod_{k=1}^{m}\left(f_{Q} w_{k} d x\right)^{\frac{p}{p_{k}}} \lesssim f \prod_{Q} \prod_{k=1}^{m} w_{k}^{\frac{p}{p_{k}}} d x .
$$

This was originally proved in the bilinear case by the first author and Neugebauer $[\mathbf{1 1}]$; for simpler proofs in the multilinear case, see $[\mathbf{9}, \mathbf{3 3}]$.

Lemma 2.1. Given $m \geq 1,1<p_{1}, \ldots, p_{m}<\infty, \frac{1}{p}=\frac{1}{p_{1}}+\cdots+\frac{1}{p_{m}}$, if $w_{1}, \ldots, w_{m} \in A_{\infty}$, then $\bar{w}=\prod_{k=1}^{m} w_{k}^{\frac{p}{p_{k}}} \in A_{\infty}$.

Proof: Since each $w_{k} \in A_{\infty}$, by choosing $C$ sufficiently large and $\delta<1$ sufficiently close to 1 , we have that, for every cube $Q$ and $E \subset Q$,

$$
\frac{w_{k}(E)}{w_{k}(Q)} \leq C\left(\frac{|E|}{|Q|}\right)^{\delta}
$$


But then, if we apply Hölder's inequality and the multilinear reverse Hölder's inequality, we have that

$$
\frac{\bar{w}(E)}{\bar{w}(Q)} \lesssim \frac{\prod_{k=1}^{m}\left(\int_{E} w_{k} d x\right)^{\frac{p}{p_{k}}}}{\prod_{k=1}^{m}\left(\int_{Q} w_{k} d x\right)^{\frac{p}{p_{k}}}} \leq C\left(\frac{|E|}{|Q|}\right)^{\delta} .
$$

There is a close connection between Muckenhoupt weights and the Hardy-Littlewood maximal operator, defined by

$$
M f(x)=\sup _{Q} f_{Q}|f(y)| d y \cdot \chi_{Q}(x),
$$

where the supremum is taken over all cubes $Q$. We have that if $1<p<$ $\infty$, then the maximal operator is bounded $L^{p}(w)$ if and only if $w \in A_{p}$. Moreover, we have a weighted vector-valued inequality that generalizes the Fefferman-Stein inequality. This was first proved by Andersen and John [1]; for an elementary proof via extrapolation, see [7].

Lemma 2.2. Given $1<p, q<\infty$ and $w \in A_{p}$, then, for any sequence $\left\{f_{k}\right\}$ in $L^{p}(w)$,

$$
\left\|\left(\sum_{k}\left(M f_{k}\right)^{q}\right)^{\frac{1}{q}}\right\|_{L^{p}(w)} \lesssim\left\|\left(\sum_{k}\left|f_{k}\right|^{q}\right)^{\frac{1}{q}}\right\|_{L^{p}(w)} .
$$

Remark 2.3. Below we will repeatedly apply Lemma 2.2 in the following way. Fix $0<p<\infty$ and $w \in A_{\infty}$. Then $w \in A_{q}$ and without loss of generality we may assume $p<q$. Let $r=\frac{q}{p}>1$. Given a sequence of cubes $Q_{k}$, let $Q_{k}^{*}=\tau Q_{k}, \tau>1$. Then $\chi_{Q_{k}^{*}} \lesssim M\left(\chi_{Q_{k}}\right)$, and the implicit constant depends only on $n$ and $\tau$. But then by Lemma 2.2 we have that, for any non-negative $\lambda_{k}$,

$$
\begin{aligned}
\left\|\sum_{k} \lambda_{k} \chi_{Q_{k}^{*}}\right\|_{L^{p}(w)} & \lesssim\left\|\sum_{k} M\left(\lambda_{k}^{\frac{1}{r}} \chi_{Q_{k}}^{\frac{1}{r}}\right)^{r}\right\|_{L^{p}(w)}=\left\|\left(\sum_{k} M\left(\lambda_{k}^{\frac{1}{r}} \chi_{Q_{k}}^{\frac{1}{r}}\right)^{r}\right)^{\frac{1}{r}}\right\|_{L^{q}(w)}^{r} \\
& \lesssim\left\|\left(\sum_{k} \lambda_{k} \chi_{Q_{k}}\right)^{\frac{1}{r}}\right\|_{L^{q}(w)}^{r}=\left\|\sum_{k} \lambda_{k} \chi_{Q_{k}}\right\|_{L^{p}(w)} .
\end{aligned}
$$

Below we will need to prove a weighted norm inequality for an $m$-CZO. To do so, we will make use of some recent developments in the theory of harmonic analysis on the domination of singular integrals by sparse operators. Here we sketch the basic definitions; for further information, see, for instance, [5]. 
A collection of cubes $\mathcal{S}$ is called a sparse family if each cube $Q \in \mathcal{S}$ contains measurable subset $E_{Q} \subset Q$ such that $\left|E_{Q}\right| \geq \frac{1}{2}|Q|$ and the family $\left\{E_{Q}\right\}_{Q \in \mathcal{S}}$ is pairwise disjoint. Given a sparse family $\mathcal{S}$ we define a linear sparse operator

$$
T^{\mathcal{S}} f(x)=\sum_{Q \in \mathcal{S}} f_{Q} f(y) d y \cdot \chi_{Q}(x) .
$$

The following estimate is proved in $[\mathbf{8}, \mathbf{2 7}]$.

Proposition 2.4. If $1<q<\infty$ and $w \in A_{q}$, then, given any sparse linear operator $T^{\mathcal{S}}$,

$$
\left\|T^{\mathcal{S}} f\right\|_{L^{q}(w)}=\left\|\sum_{Q \in \mathcal{S}} f_{Q} f d y \cdot \chi_{Q}\right\|_{L^{q}(w)} \leq C[w]_{A_{q}}^{\max \left(1, \frac{1}{q-1}\right)}\|f\|_{L^{q}(w)} .
$$

In a similar way, given a sparse family $\mathcal{S}$ we define the multilinear sparse operator

$$
T^{\mathcal{S}}\left(f_{1}, \ldots, f_{m}\right)(x)=\sum_{Q \in \mathcal{S}} \prod_{k=1}^{m} f_{Q} f_{k}\left(y_{k}\right) d y_{k} \cdot \chi_{Q}(x) .
$$

The following pointwise domination theorem was proved in $[\mathbf{2 4}$, Theorem 13.2] (see also [4]).

Proposition 2.5. Let $T$ be an $m-C Z O$ whose kernel satisfies (1.1) for any $N \geq 1$. Then given any collection $f_{1}, \ldots, f_{m}$ with bounded functions of compact support, there exists $3^{n}$ sparse families $S_{j}$ such that

$$
\left|T\left(f_{1}, \ldots, f_{m}\right)(x)\right| \leq C \sum_{j=1}^{3^{n}} T^{\mathcal{S}_{j}}\left(\left|f_{1}\right|, \ldots,\left|f_{m}\right|\right)(x) .
$$

Weighted Hardy spaces. In this section we define the weighted Hardy spaces and prove a finite atomic decomposition theorem. In defining them we follow Strömberg and Torchinsky [31] and we refer the reader there for more information.

Let $\mathscr{S}\left(\mathbb{R}^{n}\right)$ denote the Schwartz class of smooth functions. For $N_{0} \in \mathbb{N}$ to be a large value determined later, define

$$
\mathfrak{F}_{N_{0}}=\left\{\varphi \in \mathscr{S}\left(\mathbb{R}^{n}\right): \int(1+|x|)^{N_{0}}\left(\sum_{|\alpha| \leq N_{0}}\left|\frac{\partial^{\alpha}}{\partial x^{\alpha}} \varphi(x)\right|^{2}\right) d x \leq 1\right\} .
$$

Fix $0<p<\infty$ and $w \in A_{\infty}$; we define the weighted Hardy space $H^{p}(w)$ to be the set of distributions

$$
H^{p}(w)=\left\{f \in \mathscr{S}^{\prime}\left(\mathbb{R}^{n}\right): \mathcal{M}_{N_{0}}(f) \in L^{p}(w)\right\}
$$


with the quasi-norm

$$
\|f\|_{H^{p}(w)}=\left\|\mathcal{M}_{N_{0}}(f)\right\|_{L^{p}(w)},
$$

where the grand maximal function $\mathcal{M}_{N_{0}}(f)$ is defined by

$$
\mathcal{M}_{N_{0}}(f)(x)=\sup _{\varphi \in \widetilde{F}_{N_{0}}} \sup _{t>0}\left|\varphi_{t} * f(x)\right| .
$$

Note that in this definition, $N_{0}$ is taken to be a large positive integer, depending on $n, p$, and $w$, whose value is chosen so that the usual definitions of unweighted Hardy spaces remain equivalent in the weighted setting. Its exact value does not matter for us.

Given an integer $N>0$, an $(N, \infty)$ atom is a function $a$ such that there exists a cube $Q$ with $\operatorname{supp}(a) \subset Q,\|a\|_{\infty} \leq 1$, and for all $|\beta| \leq N$

$$
\int_{\mathbb{R}^{n}} x^{\beta} a(x) d x=0 .
$$

In [31, Chapter VIII] it was shown that every $f \in H^{p}(w)$ has an atomic decomposition: for every $N \geq s_{w}$ there exist a sequence of nonnegative numbers $\left\{\lambda_{k}\right\}$ and a sequence of smooth $(N, \infty)$ atoms $\left\{a_{k}\right\}$ with $\operatorname{supp}\left(a_{k}\right) \subset Q_{k}$, such that

$$
f=\sum_{k} \lambda_{k} a_{k}
$$

and the sum converges in the sense of distributions and in the $H^{p}(w)$ quasi-norm. Moreover, we have that

$$
\left\|\sum_{k} \lambda_{k} \chi_{Q_{k}}\right\|_{L^{p}(w)} \lesssim\|f\|_{H^{p}(w)} .
$$

Below, we want to use the atomic decomposition to estimate the norm of an $m$-CZO. One technical obstacle, however, is that this atomic decomposition may be an infinite sum, and therefore it is not immediate that we can exchange sum and integral in the definition of an $m$-CZO. For the argument to overcome this problem in the unweighted setting, see [17]. Our approach here is different: we show that for a dense subset of $H^{p}(w)$, we can form the atomic decomposition using a finite sequence of atoms. Our result generalizes a result in the unweighted case from $[\mathbf{2 6}]$; in the weighted case it generalizes results proved in $[\mathbf{1 2}, \mathbf{2 8}]$.

To state our result, note that for $N \geq s_{w}$, if we define

$$
\mathcal{O}_{N}=\left\{f \in \mathcal{C}_{0}^{\infty}: \int_{\mathbb{R}^{n}} x^{\alpha} f(x) d x=0, \quad 0 \leq|\alpha| \leq N\right\},
$$

then $\mathcal{O}_{N} \cap H^{p}(w)$ is dense in $H^{p}(w)$. 
Theorem 2.6. Fix $w \in A_{\infty}$ and $0<p<\infty$, and let $N \geq s_{w}$. For each $f \in \mathcal{O}_{N} \cap H^{p}(w)$, there exists a finite sequence of non-negative numbers $\left\{\lambda_{k}\right\}_{k}$ and a sequence $\left\{a_{k}\right\}$ of $(N, \infty)$ atoms, $\operatorname{supp}\left(a_{k}\right) \subset Q_{k}$, such that $f=\sum_{k} \lambda_{k} a_{k}$ and

$$
\left\|\sum_{k} \lambda_{k} \chi_{Q_{k}}\right\|_{L^{p}(w)} \leq C\|f\|_{H^{p}(w)} .
$$

The proof of Theorem 2.6 is gotten by a close analysis of the atomic decomposition given above. To prove it, we use the following technical result. It is adapted from the corresponding result from [31, Chapter VIII] (in the weighted case) and from the proof of the unweighted version of Theorem 2.6 in [26]. (See also the construction of the atomic decomposition in $[\mathbf{1 2}]$.) Indeed weights play almost no role in the result except in (4).

Lemma 2.7. Fix $w \in A_{\infty}, 0<p<\infty$, and $N \geq s_{w}$, and let $f \in$ $\mathcal{O}_{N} \cap H^{p}(w)$. For each $k \in \mathbb{Z}$, let

$$
\Omega_{k}=\left\{x \in \mathbb{R}^{n}: \mathcal{M}_{N_{0}} f(x)>2^{k}\right\} .
$$

Then there exists a sequence $\left\{\beta_{k, i}\right\}$ of smooth functions with compact support and a family of cubes $\left\{Q_{k, i}\right\}$ with finite overlap such that the following hold:

(1) For each $k$ and all $i, Q_{k, i} \subset Q_{k, i}^{*} \subset \Omega_{k}$, where $Q_{k, i}^{*}=\tau Q_{k, i}$ for $a$ fix constant $\tau>1$ and the $Q_{k, i}^{*}$ also have finite overlap.

(2) The $\beta_{k, i}$ are $(N, \infty)$ atoms with $\operatorname{supp}\left(\beta_{k, i}\right) \subset Q_{k, i}^{*}$. In particular, $\sum_{i}\left|\beta_{k, i}\right| \lesssim C$ uniformly for all $k \in \mathbb{Z}$.

(3) $f=\sum_{k, i} \lambda_{k, i} \beta_{k, i}$, where the convergence is unconditional both pointwise and in the sense of distributions.

(4) $\lambda_{k, i} \lesssim 2^{k}$ for all $k$, $i$ and $\sum_{k, i} \lambda_{k, i} \chi_{Q_{k, i}} \lesssim \mathcal{M}_{N_{0}}(f)$. In particular, $\sum_{k, i} \lambda_{k, i} \beta_{k, i}$ also converges absolutely to $f$ in $L^{q}(w)$, whenever $q>$ 1 is such that $w \in A_{q}$.

Proof of Theorem 2.6: Fix $f \in \mathcal{O}_{N} \cap H^{p}(w)$; by homogeneity we may assume without loss of generality that $\|f\|_{H^{p}(w)}=1$. Then there exists $R>1$ such that $\operatorname{supp}(f) \subset B(0, R)=B$. Let $B^{*}=B(0,4 R)$. We claim that, for all $x \notin B^{*}$,

$$
\mathcal{M}_{N_{0}} f(x) \lesssim w(B)^{\frac{-1}{p}}\|f\|_{H^{p}(w)} \lesssim \frac{1}{w\left(B^{*}\right)^{\frac{1}{p}}} .
$$


To prove this, we argue as in [12, Lemma 7.11] (cf. inequality (7.7)). There they showed a pointwise inequality: given any $\varphi \in \mathfrak{F}_{N_{0}}$ and $t>0$,

$$
\left|f * \varphi_{t}(x)\right| \lesssim \inf _{z \in B_{*}} \mathcal{M}_{N_{0}} f(z),
$$

where $B_{*}=B\left(0, \frac{1}{2} R\right)$. Therefore, we have that

$$
\left|f * \varphi_{t}(x)\right|^{p} \lesssim \frac{1}{w\left(B_{*}\right)} \int_{B_{*}} \mathcal{M}_{N_{0}} f(z)^{p} w(z) d z \leq \frac{1}{w\left(B_{*}\right)}
$$

inequality (2.2) follows if we take the supremum over all $\varphi \in \mathfrak{F}_{N_{0}}$ and $t>0$, and note that since $w \in A_{\infty}, w\left(B^{*}\right) \lesssim w\left(B_{*}\right)$.

Now let $k_{0}$ be the smallest integer such that for all $k>k_{0}, \Omega_{k} \subset B^{*}$. More precisely, by $(2.2)$ we can take $k_{0}$ to be the largest integer such that $2^{k_{0}} \leq C w\left(B^{*}\right)^{\frac{-1}{p}}$.

By Lemma 2.7 we can decompose $f$ as

$$
f=\sum_{k, i} \lambda_{k, i} \beta_{k, i}
$$

where the $\beta_{k, i}$ are $(N, \infty)$ atoms. We will show that this sum can be rewritten as a finite sum of atoms. Set

$$
F_{1}=\sum_{k \leq k_{0}} \sum_{i} \lambda_{k, i} \beta_{k, i}=f-\sum_{k>k_{0}} \sum_{i} \lambda_{k, i} \beta_{k, i} .
$$

Since the $\beta_{k, i}$ are supported in $\Omega_{k} \subset B^{*}$ for all $k>k_{0}$, the function $F_{1}$ is also supported in $B^{*}$. Moreover

$$
\left\|F_{1}\right\|_{\infty} \leq \sum_{k \leq k_{0}}\left\|\sum_{i} \lambda_{k, i}\left|\beta_{k, i}\right|\right\|_{L^{\infty}} \lesssim \sum_{k \leq k_{0}} 2^{k}=C_{1} 2^{k_{0}} .
$$

Further, $F_{1}$ has vanishing moments up to order $N$. To see this, fix $|\alpha| \leq$ $N$ and $q>1$ such that $w \in A_{q}$. Then, since $\operatorname{supp}\left(\beta_{k, i}\right) \subset B^{*}$,

$$
\begin{aligned}
\left\|\sum _ { k \leq k _ { 0 } } \sum _ { i } \left|x^{\alpha}\left\|\lambda_{k, i} \beta_{k, i} \mid\right\|_{L^{1}}\right.\right. & \leq(4 R)^{|\alpha|}\left\|\sum_{k \leq k_{0}} \sum_{i}\left|\lambda_{k, i} \beta_{k, i}\right|\right\|_{L^{q}(w)} w^{1-q^{\prime}}\left(B^{*}\right)^{\frac{1}{q^{\prime}}} \\
& \lesssim(4 R)^{|\alpha|}\left\|\mathcal{M}_{N_{0}} f\right\|_{L}^{q}(w) w^{1-q^{\prime}}\left(B^{*}\right)^{\frac{1}{q^{\prime}}} \\
& \lesssim(4 R)^{|\alpha|}\|f\|_{L}^{q}(w) w^{1-q^{\prime}}\left(B^{*}\right)^{\frac{1}{q^{\prime}}}<\infty .
\end{aligned}
$$

Therefore, the series on the left-hand side converges absolutely, so you can exchange the sum and integral; since each $\beta_{k, i}$ has vanishing moments, so does $F_{1}$. Therefore, if we set $a_{0}=C_{1}^{-1} 2^{-k_{0}} F_{1}$ then $a_{0}$ is an $(N, \infty)$ atom supported in $B^{*}$. 
To estimate the remaining terms, note that $f$ is a bounded function and so there exists an integer $k_{\infty}>k_{0}$ such that $\Omega_{k}=\emptyset$ for all $k \geq k_{\infty}$. Thus the sum

$$
\sum_{k>k_{0}} \sum_{i} \lambda_{k, i} \beta_{k, i}=\sum_{k_{0}<k<k_{\infty}} \sum_{i} \lambda_{k, i} \beta_{k, i}
$$

has finite many terms under the summation of $k$ indices. Further, since the sum $\sum_{k, i} \lambda_{k, i} \chi_{Q_{k, i}} \lesssim \mathcal{M}_{N_{0}} f$ it converges everywhere. Therefore, for each $k_{0}<k<k_{\infty}$ there exists an integer $\rho_{k}$ such that

$$
\sum_{i>\rho_{k}} \lambda_{k, i}\left|\beta_{k, i}\right| \leq 2^{-k_{\infty}} w\left(B^{*}\right)^{-\frac{1}{p}}
$$

If we define

$$
F_{2}=\sum_{k_{0}<k<k_{\infty}} \sum_{i>\rho_{k}} \lambda_{k, i} \beta_{k, i},
$$

then $F_{2}$ is supported in $B^{*}$ and

$$
\left\|F_{2}\right\|_{\infty} \leq \sum_{k_{0}<k<k_{\infty}} 2^{-k_{\infty}} w\left(B^{*}\right)^{-\frac{1}{p}} \leq C_{2} w\left(B^{*}\right)^{-\frac{1}{p}} .
$$

Moreover, arguing as we did above for $F_{1}$, we have that $F_{2}$ has vanishing moments for $|\alpha| \leq N$. Thus if we set $a_{\infty}=C_{2}^{-1} w\left(B^{*}\right)^{\frac{1}{p}} F_{2}$, then $a_{\infty}$ is an $(N, \infty)$ atom.

Therefore, we have shown that we can decompose $f$ as a finite sum of $(N, \infty)$ atoms:

$$
f=\left(C_{1} 2^{k_{0}}\right) a_{0}+\sum_{k_{0}<k<k_{\infty}} \sum_{1 \leq i \leq \rho_{k}} \lambda_{k, i} \beta_{k, i}+C_{2} w\left(B^{*}\right)^{-\frac{1}{p}} a_{\infty} .
$$

It remains to prove that (2.1) holds. But by our choice of $k_{0}$, we have that $\left\|C_{1} 2^{k_{0}} \chi_{B^{*}}\right\|_{L^{p}(w)} \leq C$, and clearly $\left\|w\left(B^{*}\right)^{-\frac{1}{p}} \chi_{B^{*}}\right\|_{L^{p}(w)} \leq C$. Finally, by the weighted Fefferman-Stein inequality (see Remark 2.3), we have that

$$
\begin{aligned}
\left\|\sum_{k_{0}<k<k_{\infty}} \sum_{1 \leq i \leq \rho_{k}} \lambda_{k, i} \chi_{Q_{k, i}^{*}}\right\|_{L^{p}(w)} \lesssim\left\|\sum_{k_{0}<k<k_{\infty}} \sum_{1 \leq i \leq \rho_{k}} \lambda_{k, i} \chi_{Q_{k, i}}\right\|_{L^{p}(w)} \\
\\
\lesssim\left\|\mathcal{M}_{N_{0}} f\right\|_{L^{p}(w)} \lesssim 1 .
\end{aligned}
$$

Since $\|f\|_{H^{p}(w)}=1$, we get the desired inequality, and this completes the proof of Theorem 2.6.

\section{Auxiliary results}

In this section we state and prove several lemmas on averaging operators and $m$-CZOs needed for the proofs of Theorems 1.1 and 1.2. 
Averaging operators. We begin with a well-known result on the maximal operator $M_{\mu}$ defined with respect to a measure $\mu$ :

$$
M_{\mu} f(x)=\sup _{Q} \frac{1}{\mu(Q)} \int_{Q}|f| d \mu \cdot \chi_{Q}(x)
$$

For a proof, see [15, Chapter II].

Proposition 3.1. Let $\mu$ be a doubling measure on $\mathbb{R}^{n}$. Then the maximal operator $M_{\mu}$ satisfies the weak $(1,1)$ inequality

$$
\sup _{t>0} t \mu\left(\left\{x \in \mathbb{R}^{n}: M_{\mu} f(x)>t\right\}\right) \leq C(\mu) \int_{\mathbb{R}}^{n}|f| d \mu
$$

and for $1<p<\infty$ the strong $(p, p)$ inequality

$$
\int_{\mathbb{R}}^{n}\left(M_{\mu} f\right)^{p} d \mu \leq C(\mu, p) \int_{\mathbb{R}}^{n}|f|^{p} d \mu
$$

The next three lemmas on averaging operators are weighted extensions of results from [18]. Our proofs, however, are different and are motivated by ideas from $[\mathbf{3 1}]$.

Lemma 3.2. Let $\mu$ be a doubling measure on $\mathbb{R}^{n}$ and fix $0<p<1$. Then, given any finite collection $\mathcal{J}$ of cubes and any set $\left\{f_{Q}: Q \in \mathcal{J}\right\}$ of non-negative integrable functions with $\operatorname{supp}\left(f_{Q}\right) \subset Q$,

$$
\left\|\sum_{Q \in \mathcal{J}} f_{Q}\right\|_{L^{p}(\mu)} \leq C(\mu, p, n)\left\|\sum_{Q \in \mathcal{J}} a_{1}^{\mu}(Q) \chi_{Q}\right\|_{L^{p}(\mu)},
$$

where

$$
a_{1}^{\mu}(Q)=\mu(Q)^{-1} \int_{Q} f_{Q}(x) d \mu(x)
$$

Proof: Let $F=\sum_{Q \in \mathcal{J}} f_{Q}$ and $G=\sum_{Q \in \mathcal{J}} a_{1}^{\mu}(Q) \chi_{Q}$ and for each $t>0$ let

$$
L_{t}=\left\{x \in \mathbb{R}^{n}: G(x)>t\right\}, \quad U_{t}=\left\{y \in \mathbb{R}^{n}: M_{\mu} \chi_{L_{t}}(y)>\frac{1}{4}\right\}
$$


By (3.1) we have that $\mu\left(U_{t}\right) \leq C(\mu) \mu\left(L_{t}\right)$. We can now estimate as follows:

$$
\begin{aligned}
\mu\left(\left\{x \in \mathbb{R}^{n}: F(x)>t\right\}\right) & \leq \mu\left(U_{t}\right)+\mu\left(U_{t}^{c} \cap\left\{x \in \mathbb{R}^{n}: F(x)>t\right\}\right) \\
& \lesssim \mu\left(L_{t}\right)+\frac{1}{t} \int_{U_{t}^{c}} F(x) d \mu(x) \\
& \lesssim \mu\left(L_{t}\right)+\frac{1}{t} \sum_{Q \in \mathcal{J}: Q \cap U_{t}^{c} \neq \emptyset} \int_{Q} f_{Q}(x) d \mu(x) \\
& \lesssim \mu\left(L_{t}\right)+\frac{1}{t} \sum_{Q \in \mathcal{J}: Q \cap U_{t}^{c} \neq \emptyset} a_{1}^{\mu}(Q) \mu(Q) .
\end{aligned}
$$

If $Q \in \mathcal{J}$ is such that $Q \cap U_{t}^{c} \neq \emptyset$, then $M_{\mu} \chi_{L_{t}}(z) \leq \frac{1}{4}$ for all $z \in Q \cap U_{t}^{c}$. In particular, we have

$$
\frac{\mu\left(L_{t} \cap Q\right)}{\mu(Q)} \leq \frac{1}{4},
$$

and so $\mu(Q) \leq \frac{4}{3} \mu\left(L_{t}^{c} \cap Q\right)$ for all $Q \in \mathcal{J}$. Thus we have that

$$
\begin{aligned}
\mu\left(\left\{x \in \mathbb{R}^{n}: F(x)>t\right\}\right) & \lesssim \mu\left(L_{t}\right)+\frac{1}{t} \sum_{Q \in \mathcal{J}} a_{1}^{\mu}(Q) \mu\left(Q \cap L_{t}^{c}\right) \\
& \lesssim \mu\left(L_{t}\right)+\frac{1}{t} \sum_{Q \in \mathcal{J}} a_{1}^{\mu}(Q) \int_{L_{t}^{c}} \chi_{Q}(x) d \mu(x) \\
& \lesssim \mu\left(L_{t}\right)+\frac{1}{t} \int_{L_{t}^{c}} G(x) d \mu(x) .
\end{aligned}
$$

Given this estimate, if we multiply by $p t^{p-1}$ and integrate, by Fubini's theorem we get

$$
\begin{aligned}
\|F\|_{L^{p}(\mu)}^{p}= & \int_{0}^{\infty} p t^{p-1} \mu\left(\left\{x \in \mathbb{R}^{n}: F(x)>t\right\}\right) d t \\
\lesssim & \int_{0}^{\infty} p t^{p-1} \mu\left(\left\{x \in \mathbb{R}^{n}: G(x)>t\right\}\right) d t \\
& +\int_{0}^{\infty} p t^{p-2} \int_{\left\{x \in \mathbb{R}^{n}: G(x) \leq t\right\}} G(x) d \mu(x) \\
= & \int_{\mathbb{R}}^{n} G(x)^{p} d \mu(x)+\int_{\mathbb{R}}^{n} G(x) \int_{G(x)}^{\infty} p t^{p-2} d t d \mu(x) \\
\lesssim & \|G\|_{L^{p}(\mu)}^{p} .
\end{aligned}
$$


Lemma 3.3. Let $\mu$ be a doubling measure on $\mathbb{R}^{n}$ and fix $1 \leq p<q<\infty$. Then, given any finite collection of cubes $\mathcal{J}$ and any set $\left\{f_{Q}: Q \in \mathcal{J}\right\}$ of non-negative integrable functions with $\operatorname{supp}\left(f_{Q}\right) \subset Q$,

$$
\left\|\sum_{Q \in \mathcal{J}} f_{Q}\right\|_{L^{p}(\mu)} \leq C(\mu, p, q, n)\left\|\sum_{Q \in \mathcal{J}} a_{q}^{\mu}(Q) \chi_{Q}\right\|_{L^{p}(\mu)},
$$

where

$$
a_{q}^{\mu}(Q)=\left(\frac{1}{\mu(Q)} \int_{Q}\left|f_{Q}(x)\right|^{q} d \mu(x)\right)^{\frac{1}{q}} .
$$

Proof: First suppose that $p>1$; we estimate by duality. Then there exists non-negative $g \in L^{p^{\prime}}(\mu),\|g\|_{L^{p^{\prime}(d \mu)}}=1$, such that

$$
\begin{aligned}
\left\|\sum_{Q \in \mathcal{J}} f_{Q}\right\|_{L^{p}(\mu)} & =\sum_{Q \in \mathcal{J}} \int_{Q} f_{Q}(x) g(x) d \mu(x) \\
& \leq \sum_{Q \in \mathcal{J}}\left(\int_{Q} f_{Q}(x)^{q} d \mu(x)\right)^{\frac{1}{q}}\left(\int_{Q} g(x)^{q^{\prime}} d \mu(x)\right)^{\frac{1}{q^{\prime}}} \\
& =\sum_{Q \in \mathcal{J}} a_{q}^{\mu}(Q) \mu(Q)\left[\frac{1}{\mu(Q)} \int_{Q} g^{q^{\prime}} d \mu\right]^{\frac{1}{q^{\prime}}} \\
& \leq \sum_{Q \in \mathcal{J}} a_{q}^{\mu}(Q) \int_{Q} M_{\mu}\left(g^{q^{\prime}}\right)(x)^{\frac{1}{q^{\prime}}} d \mu(x) \\
& \leq \int_{\mathbb{R}}^{n}\left[\sum_{Q \in \mathcal{J}} a_{q}^{\mu}(Q) \chi_{Q}\right] M_{\mu}\left(g^{q^{\prime}}\right)(x)^{\frac{1}{q^{\prime}}} d \mu(x) \\
& \leq\left\|\sum_{Q \in \mathcal{J}} a_{q}^{\mu}(Q) \chi_{Q}\right\|_{L^{p}(\mu)}\left\|M_{\mu}\left(g^{q^{\prime}}\right)^{\frac{1}{q^{\prime}}}\right\|_{L^{p^{\prime}}(\mu)} \\
& \leq\left\|\sum_{Q \in \mathcal{J}} a_{q}^{\mu}(Q) \chi_{Q}\right\|_{L^{p}(d \mu)}\left\|M_{\mu}\left(g^{q^{\prime}}\right)\right\|^{\frac{1}{q^{\prime}}}{ }_{L^{\frac{p^{\prime}}{q^{\prime}}}(\mu)} \\
& ;
\end{aligned}
$$

the first and third inequalities follow from Hölder's inequality, and the last from (3.2) (since $\left.p^{\prime}>q^{\prime}\right)$ and the fact that $\|g\|_{L^{p^{\prime}(\mu)}}=1$.

Finally, when $p=1$ the proof is essentially the same except that we use use the fact that $M_{\mu}$ is bounded on $L^{\infty}$. This completes the proof. 
Lemma 3.4. Let $w \in A_{\infty}$, and fix $0<p<\infty$ and $\max (1, p)<q<\infty$. Then, given any collection of cubes $\left\{Q_{k}\right\}_{k=1}^{\infty}$ and nonnegative integrable functions $\left\{g_{k}\right\}$ with $\operatorname{supp}\left(g_{k}\right) \subset Q_{k}$,

$\left\|\sum_{k=1}^{\infty} g_{k}\right\|_{L^{p}(w)} \leq C(w, p, q, n)\left\|\sum_{k=1}^{\infty}\left(\frac{1}{w\left(Q_{k}\right)} \int_{Q_{k}} g_{k}(x)^{q} w(x) d x\right)^{\frac{1}{q}} \chi_{Q_{k}}\right\|_{L^{p}(w)}$.

Proof: Since $w \in A_{\infty}$, the measure $\mu=w(x) d x$ is doubling. If $p \geq$ 1 , then if we fix an arbitrary integer $K$ and apply Lemma 3.3 to the functions $\left\{g_{k}\right\}_{k=1}^{K}$, we immediately get

$$
\left\|\sum_{k=1}^{K} g_{k}\right\|_{L^{p}(w)} \leq C(w, p, q, n)\left\|\sum_{k=1}^{K}\left(\frac{1}{w\left(Q_{k}\right)} \int_{Q_{k}} g_{k}(x)^{q} w(x) d x\right)^{\frac{1}{q}} \chi_{Q_{k}}\right\|_{L^{p}(w)} .
$$

The desired inequality now follows from Fatou's lemma.

When $0<p<1$, we can apply Lemma 3.2 to get the same conclusion, using the fact that

$$
\frac{1}{w\left(Q_{k}\right)} \int_{Q_{k}} g_{k}(x) w(x) d x \leq\left(\frac{1}{w\left(Q_{k}\right)} \int_{Q_{k}} g_{k}(x)^{q} w(x) d x\right)^{\frac{1}{q}} .
$$

Estimates for $\boldsymbol{m}$-CZOs. In this section we prove three estimates on $m$-CZOs.

Lemma 3.5. Let $T$ be the operator as in Theorem 1.1 and fix $w \in A_{q}$, $q>1$. Then, given any collection $f_{1}, \ldots, f_{m}$ of bounded functions with compact support,

$$
\left\|T\left(f_{1}, f_{2}, \ldots, f_{m}\right)\right\|_{L^{q}(w)} \leq C\left\|f_{1}\right\|_{L^{q}(w)}\left\|f_{2}\right\|_{L^{\infty}} \cdots\left\|f_{m}\right\|_{L^{\infty}} .
$$

Proof: By the domination estimate in Proposition 2.5 it will suffice to prove this estimate for any multilinear sparse operator $T^{\mathcal{S}}$ and nonnegative functions $f_{1}, \ldots, f_{m}$. By the definition of the sparse operator we have

$$
\begin{aligned}
T^{\mathcal{S}}\left(f_{1}, \ldots, f_{m}\right) \leq\left\|f_{2}\right\|_{\infty} \cdots\left\|f_{m}\right\|_{\infty} \sum_{Q \in \mathcal{S}} f_{Q} f_{1} d y \cdot \chi_{Q} & \\
& =\left\|f_{2}\right\|_{\infty} \cdots\left\|f_{m}\right\|_{\infty} T^{\mathcal{S}} f_{1},
\end{aligned}
$$

where on the right-hand side we now have a linear sparse operator. But then by Proposition 2.4 we have that

$$
\begin{aligned}
\left\|T^{\mathcal{S}}\left(f_{1}, \ldots, f_{m}\right)\right\|_{L^{q}(w)} & \lesssim\left\|T^{\mathcal{S}} f_{1}\right\|_{L^{q}(w)}\left\|f_{2}\right\|_{\infty} \cdots\left\|f_{m}\right\|_{\infty} \\
& \lesssim\left\|f_{1}\right\|_{L^{q}(w)}\left\|f_{2}\right\|_{\infty} \cdots\left\|f_{m}\right\|_{\infty} .
\end{aligned}
$$


The following lemma was first prove in [20]. For completeness we include its short proof.

Lemma 3.6. For $1 \leq k \leq m$ let $a_{k}$ be an $(N, \infty)$ atom supported in $Q_{k}$ and let $c_{k}$ be the center of $Q_{k}$. Then, given any non-empty subset $\Lambda \subset$ $\{1, \ldots, m\}$, we have that, for all $y \notin \cup_{k \in \Lambda} Q_{k}^{*}$,

$$
\left|T\left(a_{1}, \ldots, a_{m}\right)(y)\right| \lesssim \frac{\min \left\{\ell\left(Q_{k}\right): k \in \Lambda\right\}^{n+N+1}}{\left(\sum_{k \in \Lambda}\left|y-c_{k}\right|\right)^{n+N+1}} .
$$

In particular, we always have that

$$
\left|T\left(a_{1}, \ldots, a_{m}\right)\right| \chi_{\left(Q_{1}^{*} \cap \cdots \cap Q_{m}^{*}\right)^{c}} \lesssim \prod_{k=1}^{m}\left(M\left(\chi_{Q_{k}}\right)\right)^{\frac{n+N+1}{m n}} .
$$

Proof: Without loss of generality we may assume that $\Lambda=\{1, \ldots, r\}$ for some $1 \leq r \leq m$ and that

$$
\ell\left(Q_{1}\right)=\min \left\{\ell\left(Q_{k}\right): k \in \Lambda\right\} .
$$

Fix $y \notin \cup_{k \in \Lambda} Q_{k}^{*}$; because $a_{1}$ has vanishing moments up to order $N$, we can rewrite

$$
\begin{aligned}
& T\left(a_{1}, \ldots, a_{m}\right)(y)=\int_{\mathbb{R}^{m n}} K\left(y, y_{1}, \ldots, y_{m}\right) a_{1}\left(y_{1}\right) \cdots a_{m}\left(y_{m}\right) d \vec{y} \\
&= \int_{\mathbb{R}^{m n}}\left[K\left(y, y_{1}, \ldots, y_{m}\right)-P_{N}\left(y, y_{1}, y_{2}, \ldots, y_{m}\right)\right] \\
& \times a_{1}\left(y_{1}\right) \cdots a_{m}\left(y_{m}\right) d \vec{y} \\
&=\int_{\mathbb{R}^{m n}} K^{1}\left(y, y_{1}, y_{2}, \ldots, y_{m}\right) a_{1}\left(y_{1}\right) \cdots a_{m}\left(y_{m}\right) d \vec{y}
\end{aligned}
$$

where

$$
P_{N}\left(y, y_{1}, y_{2}, \ldots, y_{m}\right)=\sum_{|\alpha| \leq N} \frac{1}{\alpha !} \partial_{2}^{\alpha} K\left(y, c_{1}, y_{2}, \ldots, y_{m}\right)\left(y_{1}-c_{1}\right)^{\alpha}
$$

is the Taylor polynomial of degree $N$ of $K\left(y, \cdot, y_{2}, \ldots, y_{m}\right)$ at $c_{1}$ and

$$
K^{1}\left(y, y_{1}, \ldots, y_{m}\right)=K\left(y, y_{1}, \ldots, y_{m}\right)-P_{N}\left(y, y_{1}, y_{2}, \ldots, y_{m}\right) .
$$

By the smoothness condition of the kernel and the fact that $\left|y-y_{k}\right| \approx$ $\left|y-c_{k}\right|$ for all $k \in \Lambda$ and $y_{k} \in Q_{k}$ we have that

$$
\begin{aligned}
&\left|K\left(y, y_{1}, \ldots, y_{m}\right)-P_{N}\left(y, c_{1}, y_{2}, \ldots, y_{m}\right)\right| \\
& \lesssim\left|y_{1}-c_{1}\right|^{N+1}\left(\sum_{k \in \Lambda}\left|y-c_{k}\right|+\sum_{j=2}^{m}\left|y-y_{j}\right|\right)^{-m n-N-1} .
\end{aligned}
$$


Thus

$$
\begin{aligned}
\left|T\left(a_{1}, \ldots, a_{m}\right)(y)\right| & \lesssim \int_{\mathbb{R}^{m n}} \frac{\left|y_{1}-c_{1}\right|^{N+1}\left|a_{1}\left(y_{1}\right)\right| \cdots\left|a_{m}\left(y_{m}\right)\right|}{\left(\sum_{k \in \Lambda}\left|y-c_{k}\right|+\sum_{j=2}^{m}\left|y-y_{j}\right|\right)^{m n+N+1}} d \vec{y} \\
& \lesssim \int_{\mathbb{R}^{(m-1) n}} \frac{\ell\left(Q_{1}\right)^{n+N+1}}{\left(\sum_{k \in \Lambda}\left|y-c_{k}\right|+\sum_{j=2}^{m}\left|y_{j}\right|\right)^{m n+N+1}} d y_{2} \cdots d y_{m} \\
& \lesssim \frac{\ell\left(Q_{1}\right)^{n+N+1}}{\left(\sum_{k \in \Lambda}\left|y-c_{k}\right|\right)^{n+N+1}},
\end{aligned}
$$

which implies (3.3).

To prove (3.4), fix $y \in\left(Q_{1}^{*} \cap \cdots \cap Q_{m}^{*}\right)^{c}$; then there exists a nonempty subset $\Lambda$ of $\{1, \ldots, m\}$ such that $y \notin Q_{k}^{*}$ for all $k \in \Lambda$ and $y \in Q_{l}^{*}$ for $l \notin \Lambda$. Then by (3.3) we have that

$$
\begin{aligned}
\left|T\left(a_{1}, \ldots, a_{m}\right)(y)\right| & \lesssim \frac{\min \left\{\ell\left(Q_{k}\right): k \in \Lambda\right\}^{n+N+1}}{\left(\sum_{k \in \Lambda}\left|y-c_{k}\right|\right)^{n+N+1}} \\
& \lesssim \prod_{k \in \Lambda}\left(\frac{\ell\left(Q_{k}\right)}{\ell\left(Q_{k}\right)+\left|y-c_{k}\right|}\right)^{\frac{n+N+1}{m n}} \\
& \lesssim \prod_{k=1}^{m}\left(\frac{\ell\left(Q_{k}\right)}{\ell\left(Q_{k}\right)+\left|y-c_{k}\right|}\right)^{\frac{n+N+1}{m n}} .
\end{aligned}
$$

Inequality (3.4) follows from the definition of the maximal operator.

Lemma 3.7. Given $w \in A_{q}, 1 \leq q<\infty$, for $1 \leq k \leq m$ let $a_{k}$ be an $(N, \infty)$ atom supported in $Q_{k}$ and let $c_{k}$ be the center of $Q_{k}$. Suppose $Q_{1}$ is the cube such that $\ell\left(Q_{1}\right)=\min \left\{\ell\left(Q_{k}\right): 1 \leq k \leq m\right\}$. Then

$$
\left\|T\left(a_{1}, \ldots, a_{m}\right) \chi_{Q_{1}^{*}}\right\|_{L^{q}(w)} \lesssim w\left(Q_{1}\right)^{\frac{1}{q}} \prod_{l=1}^{m} \inf _{z \in Q_{1}} M\left(\chi_{Q_{l}}\right)(z)^{\frac{n+N+1}{m n}} .
$$

Proof: Since the $A_{p}$ classes are nested, we may assume without loss of generality that $q>1$. To prove (3.7) we consider two cases: $Q_{1}^{*} \cap Q_{k}^{*} \neq \emptyset$ for all $2 \leq k \leq m$ or this intersection is empty for at least one value of $k$. In the first case, since $\ell\left(Q_{1}\right)=\min \left\{\ell\left(Q_{k}\right): 1 \leq k \leq m\right\}$ we have $Q_{1}^{*} \subset 3 Q_{k}^{*}$ for all $1 \leq k \leq m$. This implies

$$
\inf _{z \in Q_{1}} M\left(\chi_{Q_{k}}\right)(z) \gtrsim 1
$$


for all $1 \leq k \leq m$, and so Lemma 3.5 yields

$$
\begin{aligned}
\left\|T\left(a_{1}, \ldots, a_{m}\right) \chi_{Q_{1}^{*}}\right\|_{L^{q}(w)} & \leq\left\|T\left(a_{1}, \ldots, a_{m}\right)\right\|_{L^{q}(w)} \\
& \lesssim\left\|a_{1}\right\|_{L^{q}(w)}\left\|a_{2}\right\|_{L^{\infty}} \cdots\left\|a_{m}\right\|_{L^{\infty}} \\
& \lesssim w\left(Q_{1}\right)^{\frac{1}{q}} \prod_{k=1}^{m} \inf _{z \in Q_{1}} M\left(\chi_{Q_{k}}\right)(z)^{\frac{n+N+1}{m n}} .
\end{aligned}
$$

In the second case, since $Q_{1}^{*} \cap Q_{k}^{*}=\emptyset$ for some $k$, the set

$$
\Lambda=\left\{2 \leq k \leq m: Q_{1}^{*} \cap Q_{k}^{*}=\emptyset\right\}
$$

is non-empty. Fix any point $y \in \mathbb{R}^{n}$. Then arguing as in the previous proof we have that

(3.8) $T\left(a_{1}, \ldots, a_{m}\right)(y)=\int_{\mathbb{R}^{m n}} K^{1}\left(y, y_{1}, y_{2}, \ldots, y_{m}\right) a_{1}\left(y_{1}\right) \cdots a_{m}\left(y_{m}\right) d \vec{y}$,

where $K^{1}\left(y, y_{1}, \ldots, y_{m}\right)$ is defined by (3.6). For $y_{1} \in Q_{1}$ we have that, for some $\xi_{1} \in Q_{1}$ and for all $y_{l} \in Q_{l}, 1 \leq l \leq m$,

$$
\left|K^{1}\left(y, y_{1}, \ldots, y_{m}\right)\right| \leq C \ell\left(Q_{1}\right)^{N+1}\left(\left|y-\xi_{1}\right|+\sum_{j=2}^{m}\left|y-y_{j}\right|\right)^{-m n-N-1} .
$$

For all $k \in \Lambda$, since $Q_{1}^{*} \cap Q_{k}^{*}=\emptyset,\left|y-\xi_{1}\right|+\left|y-y_{k}\right| \geq\left|\xi_{1}-y_{k}\right| \gtrsim\left|c_{1}-c_{k}\right|$. Therefore, for all $y_{1} \in Q_{1}^{*}$ and $y_{k} \in Q_{k}, k \in \Lambda$,

$$
\left|K^{1}\left(y, y_{1}, \ldots, y_{m}\right)\right| \lesssim \ell\left(Q_{1}\right)^{N+1}\left(\sum_{k \in \Lambda}\left|c_{1}-c_{k}\right|+\sum_{j=2}^{m}\left|y-y_{j}\right|\right)^{-m n-N-1}
$$

If we combine this inequality with (3.8), we get

$$
\begin{aligned}
\left|T\left(a_{1}, \ldots, a_{m}\right)(y)\right| & \lesssim \frac{\ell\left(Q_{1}\right)^{n+N+1}}{\left(\sum_{k \in \Lambda}\left|c_{1}-c_{k}\right|\right)^{n+N+1}} \\
& \lesssim \frac{\ell\left(Q_{1}\right)^{n+N+1}}{\left(\sum_{k \in \Lambda}\left[\ell\left(Q_{1}\right)+\left|c_{1}-c_{k}\right|+\ell\left(Q_{k}\right)\right]\right)^{n+N+1}}
\end{aligned}
$$

Since $Q_{1}^{*} \subset 3 Q_{l}^{*}$ for all $l \notin \Lambda$, the last inequality gives us

$$
\left\|T\left(a_{1}, \ldots, a_{m}\right)\right\|_{L^{\infty}} \lesssim \prod_{k=1}^{m} \inf _{z \in Q_{1}} M\left(\chi_{Q_{k}}\right)(z)^{\frac{n+N+1}{m n}} ;
$$

since $w \in A_{q}$ is doubling, this implies that

$$
\left\|T\left(a_{1}, \ldots, a_{m}\right) \chi_{Q_{1}^{*}}\right\|_{L^{q}(w)} \lesssim w\left(Q_{1}\right)^{\frac{1}{q}} \prod_{k=1}^{m} \inf _{z \in Q_{1}} M\left(\chi_{Q_{k}}\right)(z)^{\frac{n+N+1}{m n}} .
$$

This completes the proof. 


\section{Proof of Theorem 1.1}

For $1 \leq k \leq m$, let $w_{k} \in A_{\infty}$ and fix arbitrary functions $f_{k} \in$ $H^{p_{k}}\left(w_{k}\right) \cap \mathcal{O}_{N}\left(\mathbb{R}^{n}\right)$. By Theorem 2.6, we have the finite atomic decompositions

$$
f_{k}=\sum_{j_{k}=1}^{N_{0}} \lambda_{k, j_{k}} a_{k, j_{k}}
$$

where $\lambda_{k, j_{k}} \geq 0$ and $a_{k, j_{k}}$ are $(N, \infty)$-atoms that satisfy

$$
\operatorname{supp}\left(a_{k, j_{k}}\right) \subset Q_{k, j_{k}}, \quad\left|a_{k, j_{k}}\right| \leq \chi_{Q_{k, j_{k}}}, \quad \int_{Q_{k, j_{k}}} x^{\alpha} a_{k, j_{k}}(x) d x=0
$$

for all $|\alpha| \leq N$, and

$$
\left\|\sum_{j_{k}} \lambda_{j_{k}} \chi_{Q_{j_{k}}}\right\|_{L^{p_{k}\left(w_{k}\right)}} \leq C\left\|f_{k}\right\|_{H^{p_{k}}\left(w_{k}\right)} .
$$

Set $\bar{w}=\prod_{k=1}^{m} w_{k}^{\frac{p}{p_{k}}}$. Again by Theorem 2.6, it will suffice to prove that

$$
\left\|T\left(f_{1}, \ldots, f_{m}\right)\right\|_{L^{p}(\bar{w})} \lesssim \prod_{k=1}^{m}\left\|\sum_{j_{k}} \lambda_{k, j_{k}} \chi_{Q_{k, j_{k}}}\right\|_{L^{p_{k}}\left(w_{k}\right)} .
$$

Since $T$ is $m$-linear, we have that, for a.e. $x \in \mathbb{R}^{n}$,

$$
T\left(f_{1}, \ldots, f_{m}\right)(x)=\sum_{j_{1}} \cdots \sum_{j_{m}} \lambda_{1, j_{1}} \cdots \lambda_{m, j_{m}} T\left(a_{1, j_{1}}, \ldots, a_{m, j_{m}}\right)(x) .
$$

Given a cube $Q$, let $Q^{*}=2 \sqrt{n} Q$. For each $m$-tuple, $\left(j_{1}, \ldots, j_{m}\right)$, define $R_{j_{1}, \ldots, j_{m}}$ to be the smallest cube among $Q_{1, j_{1}}^{*}, \ldots, Q_{m, j_{m}}^{*}$. To estimate $\left\|T\left(f_{1}, \ldots, f_{m}\right)\right\|_{L^{p}(\bar{w})}$ we will split $T\left(f_{1}, \ldots, f_{m}\right)$ into two parts:

$$
\left|T\left(f_{1}, \ldots, f_{m}\right)(x)\right| \leq G_{1}(x)+G_{2}(x),
$$

where

$$
G_{1}(x)=\sum_{j_{1}} \cdots \sum_{j_{m}} \lambda_{1, j_{1}} \cdots \lambda_{m, j_{m}}\left|T\left(a_{1, j_{1}}, \ldots, a_{m, j_{m}}\right)\right| \chi_{R_{j_{1}, \ldots, j_{m}}}(x)
$$

and

$$
G_{2}(x)=\sum_{j_{1}} \cdots \sum_{j_{m}} \lambda_{1, j_{1}} \cdots \lambda_{m, j_{m}}\left|T\left(a_{1, j_{1}}, \ldots, a_{m, j_{m}}\right)\right| \chi_{\left(R_{j_{1}, \ldots, j_{m}}\right)^{c}}(x) .
$$


We first estimate $\left\|G_{2}\right\|_{L^{p}(\bar{w})}$. By (3.4) we have that

$$
\left|T\left(a_{1, j_{1}}, \ldots, a_{m, j_{m}}\right)(x)\right| \chi_{\left(R_{j_{1}, \ldots, j_{m}}\right)^{c}}(x) \lesssim \prod_{k=1}^{m} M\left(\chi_{Q_{k, j_{k}}}\right)(x)^{\frac{n+N+1}{m n}} ;
$$

thus

$$
G_{2} \lesssim \sum_{j_{1}} \cdots \sum_{j_{m}} \prod_{k=1}^{m} \lambda_{k, j_{k}} M\left(\chi_{Q_{k, j_{k}}}\right)^{\frac{n+N+1}{m n}}=\prod_{k=1}^{m}\left[\sum_{j_{k}} \lambda_{k, j_{k}} M\left(\chi_{Q_{k, j_{k}}}\right)^{\frac{n+N+1}{m n}}\right] .
$$

By condition (1.3), Hölder's inequality and the weighted Fefferman-Stein vector-valued inequality (see Remark 2.3), we get

$$
\left\|G_{2}\right\|_{L^{p}(\bar{w})} \lesssim \prod_{k=1}^{m}\left\|\sum_{j_{k}} \lambda_{k, j_{k}} \chi_{Q_{k, j_{k}}}\right\|_{L^{p_{k}\left(w_{k}\right)}}
$$

We now estimate the norm of $G_{1}$. Since $\bar{w} \in A_{\infty}$ by Lemma 2.1, we can choose $q>\max (1, p)$ such that $\bar{w} \in A_{q}$. Then by Lemma 3.5 we have that

$$
\begin{aligned}
\left(\frac{1}{\bar{w}\left(R_{j_{1}, \ldots, j_{m}}\right)} \int_{R_{j_{1}, \ldots, j_{m}}}\left|T\left(a_{1, j_{1}}, \ldots, a_{m, j_{m}}\right)\right|^{q}(x) \bar{w}(x) d x\right)^{\frac{1}{q}} & \\
& \lesssim \prod_{k=1}^{m} \inf _{z \in R_{j_{1}, \ldots, j_{m}}} M\left(\chi_{Q_{k, j_{k}}}\right)(z)^{\frac{n+N+1}{m n}} .
\end{aligned}
$$

If we combine this inequality, Lemma 3.4, Hölder's inequality, and the Fefferman-Stein vector-valued inequality (again see Remark 2.3), we get the following estimate:

$$
\begin{aligned}
\left\|G_{1}\right\|_{L^{p}(\bar{w}) \lesssim} & \| \sum_{j_{1} \ldots, j_{m}} \prod_{k=1}^{m} \lambda_{k, j_{k}}\left(\frac{1}{\bar{w}\left(R_{j_{1}, \ldots, j_{m}}\right)}\right. \\
& \left.\times \int_{R_{j_{1}, \ldots, j_{m}}}\left|T\left(a_{1, j_{1}}, \ldots, a_{m, j_{m}}\right)\right|^{q}(x) \bar{w}(x) d x\right)^{\frac{1}{q}} \chi_{R_{j_{1}, \ldots, j_{m}}} \|_{L^{p}(\bar{w})} \\
\lesssim & \| \sum_{j_{1} \ldots, j_{m}}\left(\prod_{k=1}^{m} \lambda_{k, j_{k}}\right) \\
& \times\left(\prod_{k=1}^{m} \inf _{z \in R_{j_{1}, \ldots, j_{m}}} M\left(\chi_{Q_{k, j_{k}}}\right)(z)^{\frac{n+N+1}{m n}}\right) \chi_{R_{j_{1}, \ldots, j_{m}}} \|_{L^{p}(\bar{w})} \\
\lesssim & \left\|\prod_{k=1}^{m}\left(\sum_{j_{k}} \lambda_{k, j_{k}} M\left(\chi_{Q_{k, j_{k}}}\right)^{\frac{n+N+1}{m n}}\right)\right\|_{L^{p}(\bar{w})}
\end{aligned}
$$




$$
\begin{aligned}
& \lesssim \prod_{k=1}^{m}\left\|\sum_{j_{k}} \lambda_{k, j_{k}} M\left(\chi_{Q_{k, j_{k}}}\right)^{\frac{n+N+1}{m n}}\right\|_{L^{p_{k}\left(w_{k}\right)}} \\
& \lesssim \prod_{k=1}^{m}\left\|\sum_{j_{k}} \lambda_{k, j_{k}} \chi_{Q_{k, j_{k}}}\right\|_{L^{p_{k}\left(w_{k}\right)}} .
\end{aligned}
$$

If we combine the estimates for $G_{1}$ and $G_{2}$, we get the desired inequality.

\section{Proof of Theorem 1.2}

The proof of Theorem 1.2 is very similar to the proof of Theorem 1.1. Instead of estimating the norm of $T$, we will estimate the norm of $M_{\phi} \circ T$, where $M_{\phi}$ is the non-tangential maximal operator

$$
M_{\phi} f(x)=\sup _{0<t<\infty} \sup _{|y-x|<t}\left|\phi_{t} * f(y)\right|
$$

where $\phi \in C_{0}^{\infty}$ and $\operatorname{supp}(\phi) \subset B(0,1)$. We will use the that the Hardy space can be characterized by using the non-tangential maximal function $M_{\phi}$ with the norm

$$
\|f\|_{H^{p}(w)} \approx\left\|M_{\phi} f\right\|_{L^{p}(w)} .
$$

See $[\mathbf{3 1}]$; this equivalence is guaranteed by our choice of $N_{0}$ sufficiently large. Throughout this section we fix a choice of $\phi$.

In this section, we fix the smooth approximate identity $\phi$ supported in the unit ball. The following lemma was first proved in [20]; it is the essential part in the proof of Theorem 1.2 and so we repeat the proof here for the convenience of the reader. Hereafter, given a cube $Q$, let $Q^{* *}=4 n Q$.

Lemma 5.1. For $1 \leq k \leq m$, let $a_{k}$ be $(N, \infty)$ atoms with $\operatorname{supp}\left(a_{k}\right) \subset$ $Q_{k}$. Suppose that $Q_{1}$ is such that $\ell\left(Q_{1}\right)=\min \left\{\ell\left(Q_{k}\right): 1 \leq k \leq m\right\}$. Then, for all $x \notin Q_{1}^{* *}$, we have

$$
\begin{aligned}
M_{\phi} T\left(a_{1}, \ldots, a_{m}\right) & (x) \lesssim \prod_{l=1}^{m} M\left(\chi_{Q_{l}}\right)(x)^{\frac{n+N+1}{m n}} \\
& +M\left(\chi_{Q_{1}}\right)(x)^{\frac{n+s_{\bar{w}}+1}{n}} \prod_{l=1}^{m} \inf _{z \in Q_{1}} M\left(\chi_{Q_{l}}\right)(z)^{\frac{N-s_{\bar{w}}}{m n}}
\end{aligned}
$$

where $T$ is the operator in Theorem 1.1. 
Proof: Fix $x \in\left(Q_{1}^{* *}\right)^{c}, 0<t<\infty$, and $y \in \mathbb{R}^{n}$ such that $|y-x|<t$. To prove (5.1) it will suffice to show that

$$
\begin{aligned}
\left|\phi_{t} * T\left(a_{1}, \ldots, a_{m}\right)(y)\right| & \lesssim \prod_{l=1}^{m} M\left(\chi_{Q_{l}}\right)(x)^{\frac{n+N+1}{m n}} \\
& +M\left(\chi_{Q_{1}}\right)(x)^{\frac{n+s_{\bar{w}}+1}{n}} \prod_{l=1}^{m} \inf _{z \in Q_{1}} M\left(\chi_{Q_{l}}\right)(z)^{\frac{N-s_{\bar{w}}}{m n}}
\end{aligned}
$$

where the implicit constant does not depend on $x, y$, and $t$. We will consider two cases.

Case 1: $t>\frac{1}{1000 n^{2}}\left|x-c_{1}\right|$. We will exploit the cancellation in (1.2) to show that

$(5.2)\left|\phi_{t} * T\left(a_{1}, \ldots, a_{m}\right)(y)\right| \lesssim M\left(\chi_{Q_{1}}\right)(x)^{\frac{n+s_{\bar{w}}+1}{n}} \prod_{l=1}^{m} \inf _{z \in Q_{1}} M\left(\chi_{Q_{l}}\right)(z)^{\frac{N-s_{\bar{w}}}{m n}}$

By (1.2) we have

$$
\begin{aligned}
& \phi_{t} * T\left(a_{1}, \ldots, a_{m}\right)(y)=\int \phi_{t}(y-z) T\left(a_{1}, \ldots, a_{m}\right)(z) d z \\
= & \int\left(\phi_{t}(y-z)-\sum_{|\alpha| \leq s_{\bar{w}}} \frac{\partial^{\alpha}\left[\phi_{t}\right]\left(y-c_{1}\right)}{\alpha !}\left(c_{1}-z\right)^{\alpha}\right) T\left(a_{1}, \ldots, a_{m}\right)(z) d z .
\end{aligned}
$$

Note that, by Taylor's theorem,

$$
\left|\phi_{t}(y-z)-\sum_{|\alpha| \leq s_{\bar{w}}} \frac{\partial^{\alpha}\left[\phi_{t}\right]\left(y-c_{1}\right)}{\alpha !}\left(c_{1}-z\right)^{\alpha}\right| \lesssim \frac{\left|z-c_{1}\right|^{s_{\bar{w}}+1}}{t^{n+s_{\bar{w}}+1}}
$$

for all $y, z \in \mathbb{R}^{n}$ and all $t \in(0, \infty)$. Since $t \gtrsim\left|x-c_{1}\right|$ and $x \notin Q_{1}^{* *}$, we have

$$
\begin{array}{rl}
\mid \phi_{t} & * T\left(a_{1}, \ldots, a_{m}\right)(y)\left|\lesssim \int \frac{\left|z-c_{1}\right|^{s_{\bar{w}}+1}}{t^{n+s_{\bar{w}}+1}}\right| T\left(a_{1}, \ldots, a_{m}\right)(z) \mid d z \\
& \lesssim\left(\frac{\ell\left(Q_{1}\right)}{\left|x-c_{1}\right|}\right)^{n+s_{\bar{w}}+1} \frac{1}{\ell\left(Q_{1}\right)^{n+s_{\bar{w}}+1}} \int\left|z-c_{1}\right|^{s_{\bar{w}}+1}\left|T\left(a_{1}, \ldots, a_{m}\right)(z)\right| d z \\
& \lesssim M\left(\chi_{Q_{1}}\right)(x)^{\frac{n+s_{\bar{w}}+1}{n}} \frac{1}{\ell\left(Q_{1}\right)^{n+s_{\bar{w}}+1}} \int\left|z-c_{1}\right|^{s_{\bar{w}}+1}\left|T\left(a_{1}, \ldots, a_{m}\right)(z)\right| d z .
\end{array}
$$


Hence, to prove (5.2) it remains to show that

$$
\begin{aligned}
\frac{1}{\ell\left(Q_{1}\right)^{n+s_{\bar{w}}+1}} \int\left|z-c_{1}\right|^{s_{\bar{w}}+1}\left|T\left(a_{1}, \ldots, a_{m}\right)(z)\right| d z \\
\\
\lesssim \prod_{l=1}^{m} \inf _{z \in Q_{1}} M\left(\chi_{Q_{l}}\right)(z)^{\frac{N-s_{\bar{w}}}{m n}}
\end{aligned}
$$

If we split the integral on the left-hand side of (5.3) over $Q_{1}^{*}$ and $\left(Q_{1}^{*}\right)^{c}$, we can estimate as follows:

$$
\begin{aligned}
\int\left|z-c_{1}\right|^{s_{\bar{w}}+1} \mid & T\left(a_{1}, \ldots, a_{m}\right)(z) \mid d z \\
\lesssim & \int_{Q_{1}^{*}}\left|z-c_{1}\right|^{s_{\bar{w}}+1}\left|T\left(a_{1}, \ldots, a_{m}\right)(z)\right| d z \\
& +\int_{\left(Q_{1}^{*}\right)^{c}}\left|z-c_{1}\right|^{s_{\bar{w}}+1}\left|T\left(a_{1}, \ldots, a_{m}\right)(z)\right| d z \\
\lesssim & \ell\left(Q_{1}\right)^{s_{\bar{w}}+1} \int_{Q_{1}^{*}}\left|T\left(a_{1}, \ldots, a_{m}\right)(z)\right| d z \\
& +\int_{\left(Q_{1}^{*}\right)^{c}}\left|z-c_{1}\right|^{s_{\bar{w}}+1}\left|T\left(a_{1}, \ldots, a_{m}\right)(z)\right| d z
\end{aligned}
$$

By (3.7), we can estimate the first integral in the last inequality by

$$
\begin{aligned}
\ell\left(Q_{1}\right)^{s \bar{w}+1} \int_{Q_{1}^{*}}\left|T\left(a_{1}, \ldots, a_{m}\right)(z)\right| d z & \\
& \lesssim \ell\left(Q_{1}\right)^{n+s \bar{w}+1} \prod_{l=1}^{m} \inf _{z \in Q_{1}} M\left(\chi_{Q_{l}}\right)(z)^{\frac{n+N+1}{m n}}
\end{aligned}
$$

To estimate the second integral, we need to exploit carefully the smoothness of the kernel. Recall the representation of $T\left(a_{1}, \ldots, a_{m}\right)(z)$ in $(3.8)$. Denote

$$
J=\left\{2 \leq l \leq m: Q_{1}^{* *} \cap Q_{l}^{* *}=\emptyset\right\} .
$$

For $z \notin Q_{1}^{*}, \xi_{1} \in Q_{1}$, we have $\left|z-\xi_{1}\right| \approx\left|z-c_{1}\right| \geq \ell\left(Q_{1}\right)$. Also, for $l \in J$ and $z_{l} \in Q_{l}^{*}$,

$$
\left|z-\xi_{1}\right|+\left|z-z_{l}\right| \geq\left|\xi_{1}-z_{l}\right| \gtrsim\left|c_{1}-c_{l}\right| .
$$


We now estimate $K^{1}\left(z, z_{1}, \ldots, z_{m}\right)$ in $(3.9)$ to get

$$
\begin{aligned}
& \left|T\left(a_{1}, \ldots, a_{m}\right)(z)\right| \\
\lesssim & \int_{\left(\mathbb{R}^{n}\right)^{m}} \frac{\ell\left(Q_{1}\right)^{N+1} \chi_{Q_{1}}\left(z_{1}\right) d z_{1} \cdots d z_{m}}{\left(\ell\left(Q_{1}\right)+\left|z-c_{1}\right|+\sum_{l \in J}\left|c_{1}-c_{l}\right|+\sum_{l=2}^{m}\left|z-z_{l}\right|\right)^{m n+N+1}}
\end{aligned}
$$

for all $z \in\left(Q_{1}^{*}\right)^{c}$. Thus

$$
\begin{aligned}
\int_{\left(Q_{1}^{*}\right)^{c}} & \left|y-c_{1}\right|^{s \bar{w}+1}\left|T\left(a_{1}, \ldots, a_{m}\right)(y)\right| d y \\
& \lesssim \int_{\mathbb{R}^{n} \times\left(\mathbb{R}^{n}\right)^{m}} \frac{\left|y-c_{1}\right|^{s \bar{w}+1} \ell\left(Q_{1}\right)^{N+1} \chi_{Q_{1}}\left(y_{1}\right) d y_{1} \cdots d y_{m} d y}{\left(\ell\left(Q_{1}\right)+\left|y-c_{1}\right|+\sum_{l \in J}\left|c_{1}-c_{l}\right|+\sum_{l=2}^{m}\left|y-y_{l}\right|\right)^{m n+N+1}} \\
& \lesssim \ell\left(Q_{1}\right)^{n+s_{\bar{w}}+1} \prod_{l \in J}\left(\frac{\ell\left(Q_{l}\right)}{\ell\left(Q_{1}\right)+\left|c_{1}-c_{l}\right|}\right)^{\frac{N-s_{\bar{w}}}{m}} .
\end{aligned}
$$

Note that $1 \lesssim \inf _{z \in Q_{1}} M\left(\chi_{Q_{l}}\right)(z)$ if $Q_{1}^{* *} \cap Q_{l}^{* *} \neq \emptyset$ and, for all $l \in J$,

$$
\frac{\ell\left(Q_{l}\right)}{\ell\left(Q_{1}\right)+\left|c_{1}-c_{l}\right|} \lesssim \inf _{z \in Q_{1}} M\left(\chi_{Q_{l}}\right)(z)^{\frac{1}{n}} .
$$

Therefore

$$
\begin{aligned}
\int_{\left(Q_{1}^{*}\right)^{c}}\left|y-c_{1}\right|^{s \bar{w}+1} \mid T & \left(a_{1}, \ldots, a_{m}\right)(y) \mid d y \\
& \lesssim \ell\left(Q_{1}\right)^{n+s \bar{w}+1} \prod_{l=1}^{m} \inf _{z \in Q_{1}} M\left(\chi_{Q_{l}}\right)(z)^{\frac{N-s_{\bar{w}}}{m n}} .
\end{aligned}
$$

Now we combine (5.4) and (5.5) we get (5.3), which completes the proof of Case 1.

Case 2: $t \leq \frac{1}{1000 n^{2}}\left|x-c_{1}\right|$. In this case, we will show that

$$
\left|\phi_{t} * T\left(a_{1}, \ldots, a_{m}\right)(y)\right| \lesssim \prod_{l=1}^{m} M\left(\chi_{Q_{l}}\right)(x)^{\frac{n+N+1}{m n}} .
$$

Since $\operatorname{supp}(\phi) \subset B(0,1)$ and $|y-x|<t$,

$$
\begin{aligned}
\left|\phi_{t} * T\left(a_{1}, \ldots, a_{m}\right)(y)\right| & \leq \int_{B(y, t)} t^{-n}\left|\phi\left(t^{-1}(y-z)\right) T\left(a_{1}, \ldots, a_{m}\right)(z)\right| d z \\
& \lesssim \sup _{z \in B(y, t)}\left|T\left(a_{1}, \ldots, a_{m}\right)(z)\right| \\
& \lesssim \sup _{z \in B(x, 2 t)}\left|T\left(a_{1}, \ldots, a_{m}\right)(z)\right|
\end{aligned}
$$


Let $\Lambda=\left\{1 \leq l \leq m: x \notin Q_{k}^{* *}\right\}$. For $z \in B(x, 2 t), \xi_{1} \in Q_{1}$, we have

$$
\begin{aligned}
\left|x-c_{1}\right| \leq|x-z|+\left|z-c_{1}\right| & \leq 2 t+\left|\xi_{1}-c_{1}\right|+\left|z-\xi_{1}\right| \\
& \leq \frac{1}{500 n^{2}}\left|x-c_{1}\right|+\frac{1}{2}\left|x-c_{1}\right|+\left|z-\xi_{1}\right| ;
\end{aligned}
$$

hence

$$
t \lesssim\left|x-c_{1}\right| \lesssim\left|z-\xi_{1}\right|
$$

For $l \in \Lambda$ and $z_{l} \in Q_{l}$, since $x \notin Q_{l}^{* *}$,

$\left|x-c_{l}\right| \leq 2\left|x-z_{l}\right| \leq 2|x-z|+2\left|z-z_{l}\right| \leq 4 t+2\left|z-z_{k}\right| \lesssim\left|z-\xi_{1}\right|+\left|z-z_{k}\right|$.

Recall the formula for $T\left(a_{1}, \ldots, a_{m}\right)(z)$ in (3.8); we estimate $K^{1}\left(z, z_{1}, \ldots\right.$, $\left.z_{m}\right)$ in (3.9) to get

$$
\left|T\left(a_{1}, \ldots, a_{m}\right)(z)\right| \lesssim \int_{\left(\mathbb{R}^{n}\right)^{m}} \frac{\ell\left(Q_{1}\right)^{N+1} \chi_{Q_{1}}\left(z_{1}\right) d z_{1} \cdots d z_{m}}{\left(\sum_{l=2}^{m}\left|z-z_{l}\right|+\sum_{k \in \Lambda}\left|x-c_{k}\right|\right)^{m n+N+1}}
$$

for all $z \in B(x, 2 t)$. From this we get that

$$
\begin{aligned}
\sup _{z \in B(x, 2 t)}\left|T\left(a_{1}, \ldots, a_{m}\right)(z)\right| & \lesssim \prod_{l \in \Lambda} \frac{\ell\left(Q_{l}\right)^{\frac{n+N+1}{|\Lambda|}} \chi_{\left(Q_{l}^{* *}\right)^{c}}(x)}{\left|x-c_{l}\right|^{\frac{n+N+1}{|\Lambda|}}} \prod_{k \notin \Lambda} \chi_{Q_{k}^{* *}}(x) \\
& \lesssim \prod_{l=1}^{m} M\left(\chi_{Q_{l}}\right)(x)^{\frac{n+N+1}{m n}} .
\end{aligned}
$$

Combining (5.7) and (5.8) gives (5.6). This completes Case 2 and so completes the proof.

The next lemma is an immediate consequence of Lemma 3.7 and the fact that $M_{\phi}$ is bounded on $L^{q}(w)$ if $w \in A_{q}$ (since it is controlled pointwise by the Hardy-Littlewood maximal operator; cf. [15]).

Lemma 5.2. Given $w \in A_{q}, 1 \leq q<\infty$, for $1 \leq k \leq m$ let $a_{k}$ be an $(N, \infty)$ atom supported in $Q_{k}$. Suppose $Q_{1}$ is the cube such that $\ell\left(Q_{1}\right)=\min \left\{\ell\left(Q_{k}\right): 1 \leq k \leq m\right\}$. Then

$$
\left\|M_{\phi} T\left(a_{1}, \ldots, a_{m}\right) \chi_{Q_{1}^{* *}}\right\|_{L^{q}(w)} \lesssim w\left(Q_{1}\right)^{\frac{1}{q}} \prod_{l=1}^{m} \inf _{z \in Q_{1}} M\left(\chi_{Q_{l}}\right)(z)^{\frac{n+N+1}{m n}} .
$$

Proof of Theorem 1.2: Fix $w_{k} \in A_{\infty}, 1 \leq k \leq m$, and define $\bar{w}=$ $\prod_{k=1}^{m} w_{k}^{\frac{p}{p_{k}}}$. Fix $f_{k} \in H^{p_{k}}\left(w_{k}\right) \cap \mathcal{O}_{N}\left(\mathbb{R}^{n}\right), 1 \leq k \leq m$. We will show that

$$
\left\|M_{\phi} T\left(f_{1}, \ldots, f_{m}\right)\right\|_{L^{p}(\bar{w})} \lesssim\left\|f_{1}\right\|_{H^{p_{1}}\left(w_{1}\right)} \cdots\left\|f_{m}\right\|_{H^{p^{m}}\left(w_{m}\right)} .
$$


Form the atomic decompositions of the functions $f_{k}$ as in the proof of Theorem 1.1 to get (4.1) and (4.2). Then to prove (5.9), it is enough to show that

$$
\left\|M_{\phi} T\left(f_{1}, \ldots, f_{m}\right)\right\|_{L^{p}(\bar{w})} \lesssim \prod_{k=1}^{m}\left\|\sum_{j_{k}} \lambda_{k, j_{k}} \chi_{Q_{k, j_{k}}}\right\|_{L^{p_{k}\left(w_{k}\right)}} .
$$

Since $M_{\phi} \circ T$ is multi-sublinear, we can write

$$
M_{\phi} T\left(f_{1}, \ldots, f_{m}\right)(x) \leq G_{1}(x)+G_{2}(x),
$$

where

$$
G_{1}(x)=\sum_{j_{1}} \cdots \sum_{j_{m}} \lambda_{1, j_{1}} \ldots \lambda_{m, j_{m}} M_{\phi} T\left(a_{1, j_{1}}, \ldots, a_{m, j_{m}}\right) \chi_{R_{j_{1}, \ldots, j_{m}}}(x)
$$

and

$$
G_{2}(x)=\sum_{j_{1}} \cdots \sum_{j_{m}} \lambda_{1, j_{1}} \cdots \lambda_{m, j_{m}} M_{\phi} T\left(a_{1, j_{1}}, \ldots, a_{m, j_{m}}\right) \chi_{\left(R_{j_{1}, \ldots, j_{m}}\right)^{c}}(x) .
$$

Here $R_{j_{1}, \ldots, j_{m}}$ is the smallest cube among $Q_{1, j_{1}}^{* *}, \ldots, Q_{m, j_{m}}^{* *}$.

A similar argument as in the proof of Theorem 1.1 with Lemma 5.2 in place of Lemma 3.7 gives

$$
\left\|G_{1}\right\|_{L^{p}(\bar{w})} \lesssim \prod_{k=1}^{m}\left\|\sum_{j_{k}} \lambda_{k, j_{k}} \chi_{Q_{k, j_{k}}}\right\|_{L^{p_{k}\left(w_{k}\right)}} .
$$

We now estimate the norm of $G_{2}$. By Lemma 5.1 we get that

$$
G_{2}(x) \lesssim G_{21}(x)+G_{22}(x),
$$

where

$$
G_{21}(x)=\sum_{j_{1}} \cdots \sum_{j_{m}} \lambda_{1, j_{1}} \cdots \lambda_{m, j_{m}} \prod_{k=1}^{m} M\left(\chi_{Q_{k, j_{k}}}\right)(x)^{\frac{n+N+1}{m n}}
$$

and

$$
\begin{array}{rl}
G_{22}(x)=\sum_{j_{1}} \cdots \sum_{j_{m}} \lambda_{1, j_{1}} \cdots \lambda_{m, j_{m}} & M\left(\chi_{R_{j_{1}, \ldots, j_{m}}}\right)(x)^{\frac{n+s_{\bar{w}}+1}{n}} \\
& \times \prod_{l=1}^{m} \inf _{z \in R_{j_{1}, \ldots, j_{m}}} M\left(\chi_{Q_{l, j_{l}}}\right)(z)^{\frac{N-s_{\bar{w}}}{m n}} .
\end{array}
$$

The function $G_{21}$ can be estimated by essentially the same argument used for $G_{1}$ to get

$$
\left\|G_{21}\right\|_{L^{p}(\bar{w})} \lesssim \prod_{k=1}^{m}\left\|\sum_{j_{k}} \lambda_{k, j_{k}} \chi_{Q_{k, j_{k}}}\right\|_{L^{p_{k}\left(w_{k}\right)}} .
$$


To estimate $G_{22}$, since $\frac{(n+s \bar{w}+1) p}{n}>1$, we use (1.4) and the FeffermanStein vector-valued inequality (cf. Remark 2.3) to get

$$
\begin{aligned}
\left\|G_{22}\right\|_{L^{p}(\bar{w})} \lesssim & \| \sum_{j_{1}} \cdots \sum_{j_{m}} \lambda_{1, j_{1}} \cdots \lambda_{m, j_{m}} \chi_{R_{j_{1}, \ldots, j_{m}}} \\
& \times \prod_{l=1}^{m} \inf _{z \in R_{j_{1}, \ldots, j_{m}}} M\left(\chi_{Q_{l, j_{l}}}\right)(z)^{\frac{N-s_{\bar{w}}}{m n}} \|_{L^{p}(\bar{w})} \\
\leq & \left\|\sum_{j_{1}} \cdots \sum_{j_{m}} \lambda_{1, j_{1}} \cdots \lambda_{m, j_{m}} \prod_{k=1}^{m} M\left(\chi_{Q_{k, j_{k}}}\right)^{\frac{N-s_{\bar{w}}}{m n}}\right\|_{L^{p}(\bar{w})} \\
\leq & \prod_{k=1}^{m}\left\|\sum_{j_{k}} \lambda_{k, j_{k}} M\left(\chi_{Q_{k, j_{k}}}\right)^{\frac{N-s_{\bar{w}}}{m n}}\right\|_{L^{p_{k}\left(w_{k}\right)}} \\
& \lesssim \prod_{k=1}^{m}\left\|\sum_{j_{k}} \lambda_{k, j_{k}} \chi_{Q_{k, j_{k}}}\right\|_{L^{p_{k}}\left(w_{k}\right)} \cdot
\end{aligned}
$$

If we combine (5.11), (5.12), and (5.13), we get (5.10) and this completes the proof.

\section{Variable Hardy spaces: Proof of Theorems 1.7 and 1.8}

In this section we prove Theorems 1.7 and 1.8. In fact, we will prove two more general results that include these theorems as special cases. To do so, we first recall some basic facts about the variable Lebsesgue spaces. For complete information we refer the reader to $[\mathbf{6}]$.

Let $\mathcal{P}_{0}\left(\mathbb{R}^{n}\right)$ be the set of all measurable functions $p(\cdot): \mathbb{R}^{n} \rightarrow(0, \infty)$. Define

$$
p_{-}=\operatorname{ess}_{x \in \mathbb{R}^{n}} p(x), \quad p_{+}=\operatorname{ess~sup}_{x \in \mathbb{R}^{n}} p(x) .
$$

Given $p(\cdot) \in \mathcal{P}_{0}\left(\mathbb{R}^{n}\right)$ define $L^{p(\cdot)}=L^{p(\cdot)}\left(\mathbb{R}^{n}\right)$ to be the set of all measurable functions $f$ such that, for some $\lambda>0$,

$$
\rho(f / \lambda)=\int_{\mathbb{R}^{n}}\left(\frac{|f(x)|}{\lambda}\right)^{p(x)} d x<\infty .
$$

This becomes a quasi-Banach space with the "norm"

$$
\|f\|_{L^{p(\cdot)}}=\inf \{\lambda>0: \rho(f / \lambda) \leq 1\} .
$$

If $p_{-} \geq 1, L^{p(\cdot)}$ is a Banach space; if $p(\cdot)=p$ a constant, then $L^{p(\cdot)}=L^{p}$ with equality of norms. 
If the maximal operator is bounded on $L^{p(\cdot)}$ we write that $p(\cdot) \in \mathcal{B}$. A necessary condition for this to be the case is that $p_{-}>1$. A sufficient condition is that $1<p_{-} \leq p_{+}<\infty$ and $p(\cdot)$ is log-Hölder continuous: i.e., (1.6) and (1.7) hold. However, this continuity condition is not necessary: see $[\mathbf{6}]$ for a detailed discussion of this problem.

Given $p(\cdot) \in \mathcal{P}_{0}\left(\mathbb{R}^{n}\right)$, the variable Hardy space $H^{p}(\cdot)$ is defined to be the set of all distributions $f$ such that $\mathcal{M}_{N_{0}} f \in L^{p(\cdot)}$. Again, we here assume $N_{0}>0$ is a sufficiently large constant so that all the standard definitions of the classical Hardy spaces are equivalent. These spaces were examined in detail in [12] (see also [28]).

A very important tool for proving norm inequalities in spaces of variable exponents is the extension of the Rubio de Francia theory of extrapolation to the scale of variable Lebesgue spaces. For the history and application of this approach for linear operators, see $[\mathbf{6}, \mathbf{7}]$. To prove Theorems 1.7 and 1.8 we will use a multilinear version due to the first author and Naibo [10]. They only stated their proof for the bilinear case, but the same proof immediately extends to the general multilinear setting.

Theorem 6.1. Let $\mathcal{F}=\left\{\left(f_{1}, \ldots, f_{m}, F\right)\right\}$ be a family of $(m+1)$-tuples of non-negative, measurable functions on $\mathbb{R}^{n}$. Suppose that there exist indices $0<p_{1}, \ldots, p_{m}, p<\infty$ satisfying $\frac{1}{p}=\frac{1}{p_{1}}+\cdots+\frac{1}{p_{m}}$ such that, for all weights $w_{k} \in A_{1}, 1 \leq k \leq m$, and $\bar{w}=\prod_{k=1}^{m} w_{k}^{\frac{p}{p_{k}}}$,

$$
\|F\|_{L^{p}(\bar{w})} \lesssim\left\|f_{1}\right\|_{L^{p_{1}}\left(w_{1}\right)} \cdots\left\|f_{m}\right\|_{L^{p_{m}}\left(w_{m}\right)}
$$

for all $\left(f_{1}, \ldots, f_{m}, F\right)$ such that $F \in L^{p}(\bar{w})$, and where the implicit constant depends only on $n, p_{k}$, and $\left[w_{k}\right]_{A_{1}}, 1 \leq k \leq m$. Let $q_{1}(\cdot), \ldots, q_{m}(\cdot)$, $q(\cdot) \in \mathcal{P}_{0}$ be such that

$$
\frac{1}{q(\cdot)}=\frac{1}{q_{1}(\cdot)}+\cdots+\frac{1}{q_{m}(\cdot)},
$$

$p_{k}<\left(q_{k}\right)_{-}, 1 \leq k \leq m$, and $q_{k}(\cdot) / p_{k} \in \mathcal{B}$. Then

$$
\|F\|_{L^{q(\cdot)}} \lesssim\left\|f_{1}\right\|_{L^{q_{1}(\cdot)}} \cdots\left\|f_{m}\right\|_{L^{q m}(\cdot)}
$$

provided $\|F\|_{L^{q(\cdot)}}<\infty$. The implicit constant only depends on $n$ and $q_{k}(\cdot)$, $1 \leq k \leq m$.

Remark 6.2 . In $[\mathbf{1 0}]$, the hypothesis on the exponents $q_{k}(\cdot)$ was stated as $\left(q_{k}(\cdot) / p_{k}\right)^{\prime} \in \mathcal{B}$, where this exponent is the conjugate exponent, defined pointwise by $\frac{1}{p(x)}+\frac{1}{p^{\prime}(x)}=1$. It was stated in this way for technical reasons related to the proof. However, these two hypotheses are equivalent: see [6, Corollary 4.64]. 
The one technical obstacle in applying Theorem 6.1 is constructing the family $\mathcal{F}$ to satisfy the hypotheses that the left-hand sides of (6.1) and (6.2) are finite and that the resulting family is large enough that the desired result can be proved via a density argument. In our case we will use the atomic decomposition in the weighted and variable Hardy spaces. As we noted in Section 2, given $w \in A_{\infty}$ and $0<p<\infty$, every $f \in H^{p}(w)$ can be written as the sum

$$
f=\sum_{k} \lambda_{k} a_{k}
$$

where $\lambda_{k} \geq 0$ and the $a_{k}$ are $(N, \infty)$ atoms, provided $N \geq s_{w}$. Moreover, this series converges both in the sense of distributions and in $H^{p}(w)$. (See [31, Chapter VIII].) The same is true in the variable Hardy spaces. More precisely: suppose $p(\cdot) \in \mathcal{P}_{0}$ is such that there exists $0<p_{0}<p_{-}$ with $p(\cdot) / p_{0} \in \mathcal{B}$. Then given $N>n\left(p_{0}^{-1}-1\right)$, if $f \in H^{p}(\cdot)$, there exists a sequence of $(N, \infty)$ atoms $a_{k}$ and constants $\lambda_{k}$ such that (6.3) holds, and the series converges both in the sense of distributions and in $H^{p}(\cdot)$. (See [12, Theorem 6.3]; here we have slightly modified the definition of atoms, but the change is immediate.) It follows immediately from these two results that finite sums of $(N, \infty)$ atoms, for $N$ sufficiently large, are dense in $H^{p}(w)$ and $H^{p}(\cdot)$.

Remark 6.3. In applying the density of finite sums of atoms, we are not making use of the finite atomic decomposition norm (as in Theorem 2.6 for weighted spaces or in the corresponding result for variable Hardy spaces in $[\mathbf{1 2}]$ ). We will only use that these sums are dense with respect to the given Hardy space norm.

Theorem 6.4. Let $q_{1}(\cdot), \ldots, q_{m}(\cdot), q(\cdot) \in \mathcal{P}_{0}$ be such that $\frac{1}{q(\cdot)}=\frac{1}{q_{1}(\cdot)}+$ $\cdots+\frac{1}{q_{m}(\cdot)}$ and $0<\left(q_{k}\right)_{-} \leq\left(q_{k}\right)_{+}<\infty, 1 \leq k \leq m$. Suppose further that there exist $0<p_{1}, \ldots, p_{m}<\infty, 0<p_{k}<\left(q_{k}\right)_{-}$, and $q_{k}(\cdot) / p_{k} \in \mathcal{B}$. If $T$ is an $m-C Z O$ as in Theorem 1.1 satisfying (1.1) for all $|\alpha| \leq N$, where

$$
N \geq \max \left\{\left\lfloor m n\left(\frac{1}{p_{k}}-1\right)\right\rfloor_{+}, 1 \leq k \leq m\right\}+(m-1) n,
$$

then

$$
T: H^{q_{1}(\cdot)} \times \cdots \times H^{q_{1}(\cdot)} \rightarrow L^{q}(\cdot) .
$$

Remark 6.5. Theorem 1.7 follows at once since if $q_{k}(\cdot)$ is log-Hölder continuous, then $q_{k}(\cdot) / p_{k} \in \mathcal{B}$. 
Proof: Fix an integer $K_{0}$ such that

$$
K_{0}>\max \left\{\left\lfloor n\left(\frac{1}{p_{k}}-1\right)\right\rfloor_{+}, 1 \leq k \leq m\right\} .
$$

Define the family $\mathcal{F}=\left\{\left(f_{1}, \ldots, f_{m}, F\right)\right\}$, where, for each $1 \leq k \leq m$,

$$
f_{k}=\sum_{j=1}^{L} \lambda_{j} a_{j}
$$

is a finite linear combination of $\left(K_{0}, \infty\right)$ atoms, and

$$
F=\min \left\{\left|T\left(f_{1}, \ldots, f_{m}\right)\right|, R\right\} \chi_{B(0, R)},
$$

where $0<R<\infty$.

Now fix any collection of weights $w_{1}, \ldots, w_{m} \in A_{1}$. Then for $1 \leq$ $k \leq m, r_{w_{k}}=1$, so $K_{0}>s_{w_{k}}$. Therefore, given any $(m+1)$-tuple $\left(f_{1}, \ldots, f_{m}, F\right) \in \mathcal{F}, f_{k} \in H^{p_{k}}\left(w_{k}\right)$, and by Theorem 1.1,

$$
\|F\|_{L^{p}(\bar{w})} \leq\left\|T\left(f_{1}, \ldots, f_{m}\right)\right\|_{L^{p}(\bar{w})} \lesssim\left\|f_{1}\right\|_{H^{p_{1}}\left(w_{1}\right)} \cdots\left\|f_{m}\right\|_{H^{p_{m}}\left(w_{m}\right)}<\infty .
$$

Moreover, we have that $f_{k} \in H^{q_{k}(\cdot)}$ and

$$
\|F\|_{q}(\cdot) \leq R\left\|\chi_{B(0, R)}\right\|_{q}(\cdot)<\infty .
$$

Hence, by Theorem 6.1 we have that

$$
\|F\|_{q(\cdot)} \lesssim\left\|f_{1}\right\|_{H^{q_{1}(\cdot)}} \cdots\left\|f_{m}\right\|_{H^{q m}(\cdot)}<\infty
$$

By Fatou's lemma in the scale of variable Lebesgue spaces [6, Theorem 2.61], we get

$$
\left\|T\left(f_{1}, \ldots, f_{m}\right)\right\|_{q(\cdot)} \lesssim\left\|f_{1}\right\|_{H^{q_{1}(\cdot)}} \cdots\left\|f_{m}\right\|_{H^{q m}(\cdot)}<\infty .
$$

Since finite sums of $\left(K_{0}, \infty\right)$ atoms are dense in $H^{q_{k}(\cdot)}, 1 \leq k \leq m$, a standard density argument shows that this inequality holds for all $f_{k} \in H^{q_{k}(\cdot)}, 1 \leq k \leq m$. This completes the proof.

The proof of the following result is identical to the proof of Theorem 6.4 , except that in the definition of the family $\mathcal{F}$ we replace $T$ by $\mathcal{M}_{N_{0}} T$ (for $N_{0}$ sufficiently large) and use Theorem 1.2 instead of Theorem 1.1. Theorem 1.8 again follows as an immediate corollary. 
Theorem 6.6. Given $q_{1}(\cdot), \ldots, q_{m}(\cdot), q(\cdot)$ and $p_{1}, \ldots, p_{m}$ as in Theorem 6.4 , define $p$ by

$$
\frac{1}{p}=\frac{1}{p_{1}}+\cdots+\frac{1}{p_{m}}
$$

Let $T$ be an $m$-CZO as in Theorem 1.1 satisfying (1.1) for all $|\alpha| \leq N$, where

$$
N \geq\left\lfloor m n\left(\frac{1}{p}-1\right)\right\rfloor_{+}+\max \left\{\left\lfloor m n\left(\frac{1}{p_{k}}-1\right)\right\rfloor_{+}, 1 \leq k \leq m\right\}+m n .
$$

Suppose further that $T$ satisfies (1.2) for all $|\alpha| \leq\lfloor n(1 / p-1)\rfloor_{+}$. Then

$$
T: H^{q_{1}(\cdot)} \times \cdots \times H^{q_{1}(\cdot)} \rightarrow H^{q}(\cdot) .
$$

\section{References}

[1] K. F. Andersen and R. T. John, Weighted inequalities for vector-valued maximal functions and singular integrals, Studia Math. 69(1) (1980/81), 19-31. DOI: 10.4064/sm-69-1-19-31.

[2] R. R. Coifman and Y. Meyer, On commutators of singular integrals and bilinear singular integrals, Trans. Amer. Math. Soc. 212 (1975), 315-331. DOI: $10.2307 / 1998628$.

[3] R. Coifman And Y. Meyer, Commutateurs d'intégrales singulières et opérateurs multilinéaires, Ann. Inst. Fourier (Grenoble) 28(3) (1978), 177-202. DOI: 10.5802/aif.708.

[4] J. M. Conde-Alonso And G Rey, A pointwise estimate for positive dyadic shifts and some applications, Math. Ann. 365(3-4) (2016), 1111-1135. DOI: $10.1007 / \mathrm{s} 00208-015-1320-y$.

[5] D. Cruz-Uribe, Two weight inequalities for fractional integral operators and commutators, in: "Proceedings of the Sixth International School of Advanced Courses of Mathematical Analysis VI" (F. J. Martín-Reyes et al., ed.), World Scientific, 2017, pp. 25-85. DOI : 10.1142/9789813147645_0002.

[6] D. V. Cruz-Uribe and A. Fiorenza, "Variable Lebesgue Spaces. Foundations and Harmonic Analysis", Applied and Numerical Harmonic Analysis, Birkhäuser/Springer, Heidelberg, 2013. DOI : 10.1007/978-3-0348-0548-3.

[7] D. V. Cruz-Uribe, J. M. Martell, and C. PÉrez, "Weights, Extrapolation and the Theory of Rubio de Francia", Operator Theory: Advances and Applications 215, Birkhäuser/Springer Basel AG, Basel, 2011. DOI : 10.1007/978-30348-0072-3.

[8] D. Cruz-Uribe, J. M. Martell, and C. Pérez, Sharp weighted estimates for classical operators, Adv. Math. 229(1) (2012), 408-441. DOI: 10.1016/j.aim. 2011.08 .013$.

[9] D. Cruz-Uribe and K. Moen, A multilinear reverse Hölder inequality with applications to multilinear weighted norm inequalities, Preprint (2017). arXiv: 1701.07800 .

[10] D. Cruz-Uribe And V. NAibo, Kato-Ponce inequalities on weighted and variable Lebesgue spaces, Differential Integral Equations 29(9-10) (2016), 801-836. 
[11] D. Cruz-Uribe and C. J. Neugebauer, The structure of the reverse Hölder classes, Trans. Amer. Math. Soc. 347(8) (1995), 2941-2960. DOI: 10.2307/ 2154763.

[12] D. Cruz-Uribe and L.-A. D. Wang, Variable Hardy spaces, Indiana Univ. Math. J. 63(2) (2014), 447-493. DOI : 10.1512/iumj .2014.63.5232.

[13] J. Duonndikoetxea, "Fourier Analysis", Translated and revised from the 1995 Spanish original by David Cruz-Uribe, Graduate Studies in Mathematics 29, American Mathematical Society, Providence, RI, 2001. DOI : 10.1090/gsm/029.

[14] J. García-Cuerva, Weighted $H^{p}$ spaces, Dissertationes Math. (Rozprawy Mat.) 162 (1979), 63 pp.

[15] J. García-Cuerva and J. L. Rubio de Francia, "Weighted Norm Inequalities and Related Topics", North-Holland Mathematics Studies 116, Notas de Matemática [Mathematical Notes] 104, North-Holland Publishing Co., Amsterdam, 1985.

[16] L. Grafakos, "Modern Fourier Analysis", Second edition, Graduate Texts in Mathematics 250, Springer, New York, 2009. DOI : 10.1007/978-0-387-09434-2.

[17] L. Grafakos and D. He, Multilinear Calderón-Zygmund operators on Hardy spaces, II, J. Math. Anal. Appl. 416(2) (2014), 511-521. DOI: 10.1016/j.jmaa. 2014.02 .051$.

[18] L. Grafakos and N. Kalton, Multilinear Calderón-Zygmund operators on Hardy spaces, Collect. Math. 52(2) (2001), 169-179.

[19] L. Grafakos and J. M. Martell, Extrapolation of weighted norm inequalities for multivariable operators and applications, J. Geom. Anal. 14(1) (2004), 19-46. DOI : $10.1007 / \mathrm{BF} 02921864$.

[20] L. Grafakos, S. Nakamura, H. V. Nguyen, and Y. Sawano, Conditions for boundedness into Hardy spaces, Math. Nachr. (to appear).

[21] L. Grafakos, S. Nakamura, H. V. Nguyen, and Y. Sawano, Multiplier conditions for boundedness into Hardy spaces, Ann. Inst. Fourier (Grenoble) (to appear).

[22] L. Grafakos and R. H. Torres, Multilinear Calderón-Zygmund theory, Adv. Math. 165(1) (2002), 124-164. DOI : 10.1006/aima.2001.2028.

[23] L. Grafakos and R. H. Torres, Maximal operator and weighted norm inequalities for multilinear singular integrals, Indiana Univ. Math. J. 51(5) (2002), 1261-1276. DOI : 10.1512/iumj.2002.51.2114.

[24] A. K. Lerner and F. NazArov, Intuitive dyadic calculus: the basics, Preprint (2015). arXiv: 1508.05639.

[25] A. K. Lerner, S. Ombrosi, C. Pérez, R. H. Torres, and R. TrujilloGonzÁlez, New maximal functions and multiple weights for the multilinear Calderón-Zygmund theory, Adv. Math. 220(4) (2009), 1222-1264. DOI: 10.1016/j.aim.2008.10.014.

[26] S. Meda, P. SJögren, and M. Vallarino, On the $H^{1}-L^{1}$ boundedness of operators, Proc. Amer. Math. Soc. 136(8) (2008), 2921-2931. DOI: 10.1090/ S0002-9939-08-09365-9.

[27] K. Moen, Sharp weighted bounds without testing or extrapolation, Arch. Math. (Basel) 99(5) (2012), 457-466. DOI : 10.1007/s00013-012-0453-4.

[28] E. NAKAI AND Y. SAWANO, Hardy spaces with variable exponents and generalized Campanato spaces, J. Funct. Anal. 262(9) (2012), 3665-3748. DOI: $10.1016 / j \cdot j f a .2012 .01 .004$. 
[29] W. OrLICZ, Über konjugierte Exponentenfolgen, Studia Math. 3 (1931), 200211. DOI : $10.4064 / \mathrm{sm}-3-1-200-211$.

[30] Y. Sawano, Atomic decompositions of Hardy spaces with variable exponents and its application to bounded linear operators, Integral Equations Operator Theory 77(1) (2013), 123-148. DOI : 10.1007/s00020-013-2073-1.

[31] J.-O. Strömberg and A. Torchinsky, "Weighted Hardy Spaces", Lecture Notes in Mathematics 1381, Springer-Verlag, Berlin, 1989. DOI: 10.1007/ BFb0091154.

[32] J. TAN, Bilinear Calderón-Zygmund operators on products of variable Hardy spaces, Forum Math. 31(1) (2019), 187-198. DOI : 10.1515/forum-2018-0082.

[33] Q. Xue AND J. YAN, Multilinear version of reversed Hölder inequality and its applications to multilinear Calderón-Zygmund operators, J. Math. Soc. Japan 64(4) (2012), 1053-1069. DOI : 10.2969/jmsj/06441053.

Department of Mathematics, University of Alabama, Tuscaloosa, AL 35487

E-mail address: dcruzuribe@ua.edu

E-mail address: kabe.moen@ua.edu

E-mail address: hvnguyen@ua.edu

Primera versió rebuda el 16 de novembre de 2017, darrera versió rebuda el 6 de març de 2018. 